NBSIR 75-968

\title{
Selected Thermochemical Data Compatible with the CODATA Recommendations
}

V. B. Parker, D. D. Wagman, D. Garvin

Physical Chemistry Division

Institute for Materials Research

National Bureau of Standards

Washington, D. C. 20234

January 1976

Interim Report

\section{Prepared for}

QC

nee:- of Standard Reference Data, NBS

100

. 456

Itional Atomic Energy Agency

no.75.968 


\section{SELECTED THERMOCHEMICAL DATA COMPATIBLE WITH THE CODATA RECOMMENDATIONS}

V. B. Parker, D. D. Wagman, D. Garvin

Physical Chemistry Division

Institute for Materials Research

National Bureau of Standards

Washington, D. C. 20234

January 1976

Interim Report

Prepared for

Office of Standard Reference Data, NBS

and

International Atomic Energy Agency

Vienna, Austria

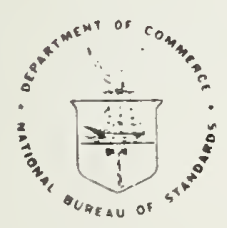

U.S. DEPARTMENT OF COMMERCE, Elliot L. Richardson, Secretary James A. Baker, III, Under Secretary

Dr. Betsy Ancker-Johnson, Assistant Secretary for Science and Technology

NATIONAL BUREAU OF STANDARDS. Ernest Ambler, Acting Director 


\section{TABLE OF CONTENTS}

1. Introduction 1

2. Contents of the Table of Thermochemical Data 2

3. Description of Entries in the Table 3

4. General Remarks 4

Table A. Physical State Conventions 6

$\begin{array}{ll}\text { Appendix. Conversion and Rounding } & 10\end{array}$

Table of Thermochemical Data 12 
This table has been designed to provide auxiliary data which may be used in the evaluation of thermochemical properties of compounds of the actinide elements. It includes the auxiliary data now being used in NBS evaluations of the properties of compounds of thorium, uranium, boron and some alkali metals. It probably is comprehensive enough for use in evaluations of compounds of the other actinides; but it can be expanded and redistributed if desirable.

The table has been prepared to assist the group of thermochemists that is evaluating the thermochemistry of the actinides for the International Atomic Energy Agency. The use of the values provided here in the preparation of each of the IAEA reviews will help assure that the values recommended in the entire set of reviews will be based on a consistent set of values for properties of compounds of the non-actinide elements.

\section{CONTENTS OF THE TABLE OF THERMOCHEMICAL DATA}

The following classes of material are given:

a) Values recommended by the CODATA Task Group as of 1975, complete [1]. The Task group recommended values for $\Delta H f(298), S(298)$ and $H(298)-H(0)$. Values listed for $\Delta G f(298)$ and $\Delta H f(0)$ have been calculated by us.

b) Properties of substances derived from measurements used in the course of the CODATA evaluations, such as properties of crystalline salts from their enthalpies and Gibbs energies of solution.

c) Recent selections for auxiliary data consistent with the CODATA Key Values that have been made by members of the Chemical Thermodynamics Data Center, NBS.

d) Selections from the NBS Technical Note 270 series [2] that have been recalculated to make them consistent with the CODATA rec ommendations.

e) Selections copied directly from the NBS Technical Note 270 series. Analysis has shown that these values are not affected by the CODATA recommendations.

For each substance in the table there is a comment identifying the source of the values except for those copies directly from the NBS Technical Note Series (class e, above). 


\section{DESCRIPTION OF ENTRIES IN THE TABLE}

A two-line entry is given for each substance. The first line contains a compound number, the molecular formula, the formula weight, and one or more of the six properties $\Delta H f(0), \Delta H f(298), \triangle G f(298)$, $\mathrm{H}(298)-\mathrm{H}(0), \mathrm{S}(298)$ and $\mathrm{Cp}(298)$ in $\mathrm{kcal} / \mathrm{mol}$ or $\mathrm{cal} / \mathrm{mol} \cdot \mathrm{K}$. The headings in the table for these properties are DHO, DH298, DG298, H298-HO, S298 and CP298, respectively. The second line shows the uncertainty for each value immediately below that entry and may have a comment in the space below the molecular formula. The symbol "+-" is used in the table for the plus or minus sign, \pm .

Because this table is based on a machine-readable data bank, certain conventions have been adopted that permit both humans and a computer to decode the material. The essential points are covered below.

a) Molecular formula. A normal single-line chemical formula is written for each substance with four modifications:

(1) Subscripts and superscripts are written on line.

(2) The subscript "1" implicit in normal chemical formulae is written explicitly wherever it is necessary to separate the symbols for two chemical elements (written entirely in capital letters): $\mathrm{CaNO}_{3}$ becomes CAIN103.

(3) The physical state of the substance is appended to the molecular formula in parentheses: HCl in 250 moles of water becomes H1CL(250H20). The conventions used in designating the state are given in Table A.

(4) Electrical charge for ions is written in the form $+\mathrm{N}$ or $-\mathrm{N}$ immediately preceding the physical state. When $\mathrm{N}=1$ the digit may be omitted.

b) Formula Weight. This is calculated from the molecular formula using the 1969 Atomic Weights [3], and is shown uniformly to four decimal places. 
c) Values for Properties. These are given in the units stated in the heading for the table, e.g. $\triangle H f(298), \triangle G f(298)$, $\mathrm{H}(298)-\mathrm{H}(0)$ and $\Delta \mathrm{Hf}(0)$ in $\mathrm{kcal} / \mathrm{mol}$, and, correspondingly, $\mathrm{S}(298)$ and $\mathrm{Cp}(298)$ in $\mathrm{ca} 1 /(\mathrm{mol} \cdot \mathrm{K})$.

Some values in the data bank are in SI and some in calorie units. When a table is prepared in a single set of units some values must be converted. Conventional conversion and rounding rules are used by the computer program. (See Appendix). These are sufficient to assure that the CODATA recommendations in SI were converted to values in calorie units that agree with the auxiliary tables in the CODATA publications. Occasionally an extra decimal place is given, but the stated value will always round to match that given by CODATA.

d) Uncertainties. These are printed immediately below the values with which they are associated. Some values in the data bank have uncertainties supplied by the evaluators; others do not. In the latter case an arbitrary value is supplied. This is "10 in the last place" as the value exists in the data bank. It appears in square brackets: $48.20+-[0.10]$, or, for a value converted from joules, $15.041+-[0.024]$, where "t-" is used for the plusor-minus sign, \pm .

\section{GENERAL REMARKS}

Suggestions for the improvement and extension of this table will be welcomed by the authors. Users are urged to report any errors and to suggest revisions in the data.

The table itself has been prepared from a machine readable data bank with the aid of a computer program that incorporates manipulations such as calculation of formula weights, conversion of units, rounding and formatting of printing. This program is a modification of one developed by Dr. J. B. Pedley, University of Sussex, England, for use in the CATCH system of themochemical analysis. 


\section{$\underline{\text { References }}$}

[1] "CODATA Recommended Key Values for Thermodynamics, 1975" (to be published as a CODATA Bulletin), and "Tentative set of Key Values for Thermodynamics: Part V' CODATA Special Report 3 , September 1975 .

[2] D. D. Wagman et. al, Nat. Bur. Stand. (U.S.) Technical Notes 270-3 (Jan. 1968), 270-4 (May 1969), 270-5 (March 1971). V. B. Parker, et. a1., NBS Technical Note 270-6 (November 1971). R. H. Schumm et al., NBS Technical Note 270-7 (April 1973).

[3] IUPAC Inorganic Chemistry Division Commission on Atomic Weights, Pure and Applied Chemistry 21, 91 (1970). 
TABLE A: Physical State Conventions

The following conventions are used to designate the physical state of a substance. These apply to the attached table and to the NBS Thermochemical Data Bank. This information appears in a parenthetical expression appended to the molecular formula. Some of the explanations imply a thermochemical value, particularly those for solutions. These normally are used in describing en thalpy mea surements.

Basic Symbols

\section{Explanation}

Gaseous, e.g., in $\mathrm{H} 1 \mathrm{CL}(\mathrm{G})$ for $\mathrm{HCl}(\mathrm{g})$

Gaseous reference standard state for an element, e.g., $02(G S)$ for $O_{2}(g)$

Crystalline, e.g., in $\mathrm{N} 1 \mathrm{H} 4 \mathrm{CL}(\mathrm{C})$ for $\mathrm{NH}_{4} \mathrm{Cl}(\mathrm{c})$ Crystalline reference standard state for an element, e.g., in $R B(C S)$ for $R b(c)$

Liquid, e.g., in $\mathrm{H} 2 \mathrm{O}(\mathrm{L})$ for $\mathrm{H}_{2} \mathrm{O}(l)$

Liquid reference standard state for an element, e.g., in $\mathrm{BR} 2(\mathrm{IS})$ for $\mathrm{Br}_{2}(l)$

Amorphous

Glassy்

Hypothetical standard state of the ideal aqueous solution at unit activity. For a neutral electrolyte the value of a property is equal to the algebraic sum of the values for the ions assumed to constitute the molecule of the electrolyte, e.g.HCl(A)= $H+(A)+C \ell-(A)$. For an tonic species this notation is commonly used to refer to the undissociated ion as written. 
Symbol

(AO)

(AU)

\section{Explanation}

Hypothetical standard state of the ideal aqueous solution at unit activity of the undissociated (non-ionized) species, e.g. $\mathrm{HF}(\mathrm{AO}), \mathrm{HF}_{2}-(\mathrm{AO})$. May also be used whenever the designation (A) could be ambiguous. Note that the descriptions $\mathrm{HSO}_{4}-(\mathrm{A})$ and $\mathrm{HSO}_{4}-(\mathrm{AO})$ are equivalent, but that $\mathrm{HF}(\mathrm{A})$ and $\mathrm{HF}(\mathrm{AO})$ are not.

Aqueous solution of undefined, but usually dilute, concentration, e.8. $\mathrm{XeO}_{3}(\mathrm{AU})$.

The symbols used above occasionally are modified by numbers to distinguish two substances in the same state that have the same molecular weight, as for isomers,: (AU2), (C3). They are also used in combination with descriptive material, e.g. (C:HE), (C:AL) etc. to mean "crystalline, hexagonal", "crystalline, alpha form" etc.

\section{Special notations for substances in solutions}

The notations for the "state" of a substance in solution may $\therefore$

combine a definition of the system, e.g. HCl in 220 moles of water, and a specification of the thermochemical property associated with it. Usually the thermochemical property is the apparent integral enthalpy or free energy of formation or an absolute entropy, i.e. the formation properties of the solvent are not included. If a partial molal property is tabulated the notation D: (" $D$ " for "differential") occurs as the first term in the state bracket. The notations given below illustrate the differences for integral and differential (partial mola1) properties, and extrapolated values. Examples are given for aqueous, mixed, and non-aqueous solvents. 
$\mathrm{H} 1 \mathrm{CL}(200 \mathrm{H} 20)$

$\mathrm{H} 1 \mathrm{CL}(\mathrm{D}: 20 \mathrm{OH} 20)$ and $\mathrm{H} 2 \mathrm{O}(\mathrm{D}: \mathrm{H} 1 \mathrm{CL}+20 \mathrm{H} 2 \mathrm{O})$
An aqueous solution of specifled composition, e.g. one mole of $\mathrm{HCl}$ in 200 moles $\mathrm{H}_{2} \mathrm{O}$. The value of $\triangle H f$ represents the apparent integral enthalpy of formation.

These represent the partial molal (enthalpy) of formation of the substance in a solution of specified concentration, e.g. the partial molal enthalpy of formation of $\mathrm{HCl}$ and $\mathrm{H}_{2} \mathrm{O}$ respectively, in a solution consisting of 1 mole $\mathrm{HCl}$ and 200 moles $\mathrm{H}_{2} \mathrm{O}$.

U1CL4(H1CL104+50H20) This describes a solute dissolved in a mixed solvent, e.g. one mole of $\mathrm{UCl}_{4}$ in a mixture of 1 mole of $\mathrm{HClO}_{4}$ and 50 moles $\mathrm{H}_{2} \mathrm{O}$. The value of $\triangle \mathrm{Hf}$ represents the apparent integral enthalpy of formation of the substance, $U C \ell_{4}$, in the medium.

U 1CI4 (H1CL104+ $50 \mathrm{H} 20: \mathrm{AU})$
This represents a solute at an unspecified but usually dilute concentration in a solvent mixture of fixed composition. 
U1CL4 (D:H1N103+50H20) Specifies the partial molal (enthalpy) of formation of a substance in a mixed medium, e.g. the enthalpy of formation of 1 mole of $\mathrm{UCl}_{4}$ in a large amount of a solution containing $\mathrm{UCl}_{4}, \mathrm{HNO}_{3}$ and $\mathrm{H}_{2} \mathrm{O}$ in the molar ratios $1: 1: 50$.

U1CI4 (L(H1CL) :AU)

Specifies a thermochemical value extrapolated from those in a particular type of solution, e.g. the integral (enthalpy) of formation of $\mathrm{UCl} l_{4}$ at an unspecified concentration in water solution obtained by extrapolation from values in solutions containing $\mathrm{HCl}$ at varying concentrations.

$\operatorname{RB} 1 I(\mathrm{C} 1 \mathrm{H} 3 \mathrm{C} 1 \mathrm{~N}: \mathrm{S})$

Ideal solution of a substance ( $R b I$ ) in a nonaqueous solvent $\left(\mathrm{CH}_{3} \mathrm{CN}\right)$, the substance being in the standard state of unit activity on the molal scale unless otherwise indicated. (In some early entries in the Data Bank these are.written ambiguously as (C1H3C1N)).

RB1I (C1H3C1N : U) Solution of a substance $(R b I)$ in a non-aqueous solvent $\left(\mathrm{CH}_{3} \mathrm{CN}\right)$ at an unspecified concentration. This is analogous to (AU).

RB1I (1000C1H3C1N) Solution of a substance (RbI) in a non-aqueous solvent $\left(\mathrm{CH}_{3} \mathrm{CN}\right)$ at a specified concentration. 
Appendix. Conversion and Rounding

The rules used here are given below. They are adaptations of rules in two editions (1970 and 1974) of the "Metric Practice Guide" published by the American Society for Testing and Materials*. The conversion procedure is from Section 4.4.1.1, ASTM E380-70 which is a special case of the procedure in Section 4.2.1, ASTM E380-74. The rounding procedure is taken without change from Section 4.4, ASTM E380-74.

We suggest that these rules are suitable for use in other applications involving the conversion of energy quantities.

1. Input to the data bank. A number and its uncertainty are written to the same least significant decimal place. Normally not more than two significant digits appear in the uncertainty.

\section{Output and Printing}

(a) A number and uncertainty present in the data bank in the same units as those used in a table are printed without modification.

(b) A number and its uncertainty, whether converted or not, are printed to the same least significant decimal place.

3. Conversion of units. The number and its uncertainty are converted separately. The decimal place at which each is to be rounded is set as follows.

(a) If the most significant digit in the converted quantity is less than the most significant digit in the original, one more significant digit is used in the converted quantity than is present in the original.

*"Standard Metric Practice Guide (A Guide to the Use of SI - the International System of Units)", ASTM Designation E380-74, American National Standard Z210.1, (American Society for Testing and Materla1s, 1916 Race Street, Philadelphia, PA. USA 19103), 1974. - , ASTM Designation E380-70, (Am. Soc. for Testing and Materials), 1970. 
Otherwise the same number of significant digits is used.

Example $2.0 \times(1 / 4.184)=0.48$

$4.3 \times(1 / 4.184)=1.03$

(b) The number of significant digits derived for the value in (a) above is decreased, if necessary, to the smaller number required by either of the following criteria.

(1) No more than two significant digits in the uncertainty, or,

(2) No more than three decimal places (0.001) in the value (and the uncertainty).

4. Rounding. Consider the quantity that is to be rounded to be written in the form N.R $\times 10^{\mathrm{K}}$

where $\mathrm{K}$ is an integer and the fractional portion $\mathrm{R}$ is to be discarded in the rounding process.

If $R<0.5$, then no change is made in $\mathrm{N}$

If $R>0.5$ then one unit is added to $\mathrm{N}$

If $R=0.5$ exactly, then one unit is added to $N$ if $N$ is odd, no change is made if $\mathrm{N}$ is even.

Note: These rules may increase the number of significant digits in a quantity, but only by one, in a sequence of conversions., e.g., joules $\rightarrow$ calories $\rightarrow$ joules $\Rightarrow$ calories, etc. 


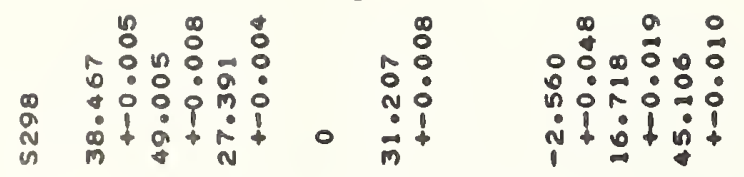

$$
\begin{aligned}
& \text { 管 }
\end{aligned}
$$

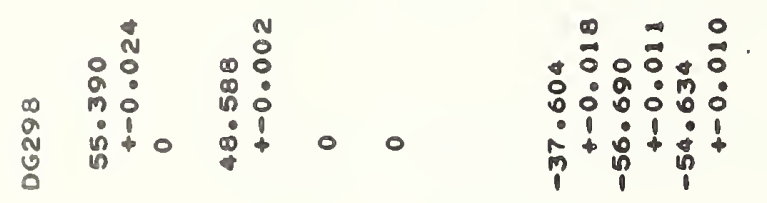

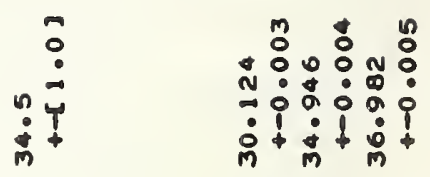
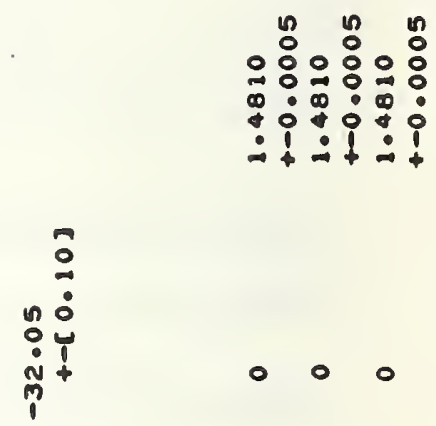

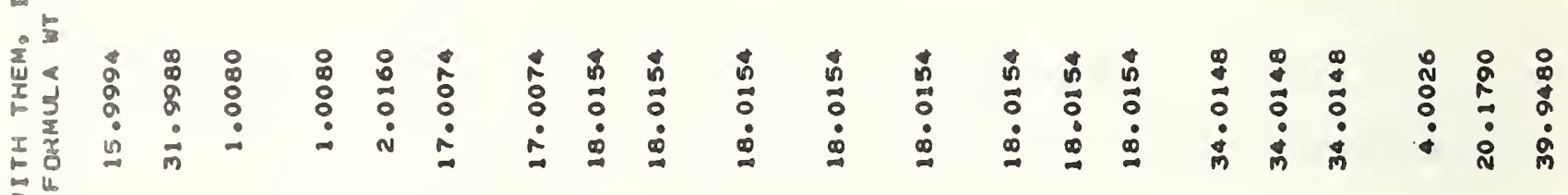




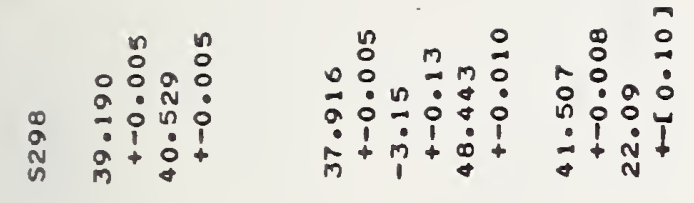

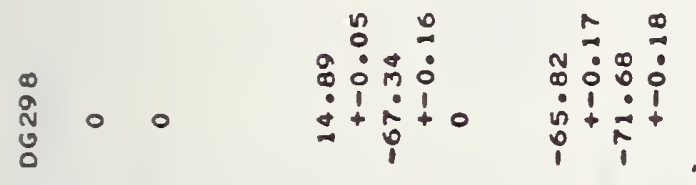

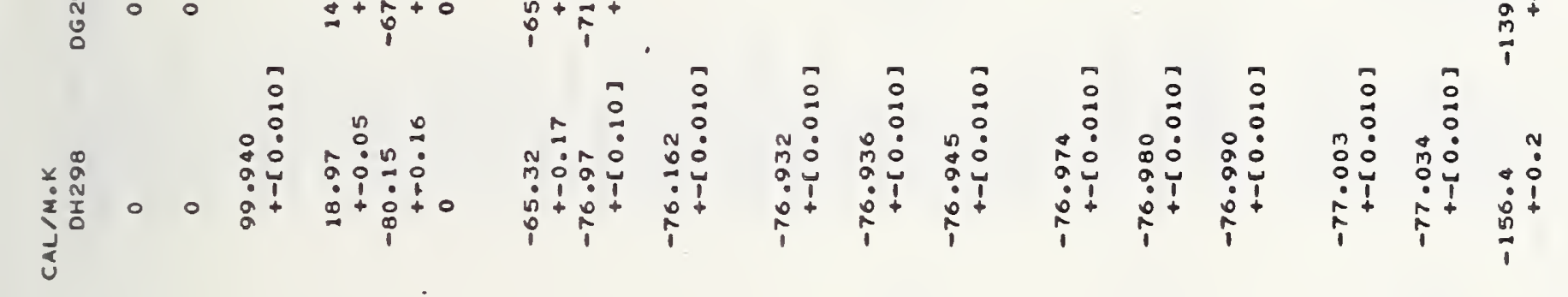

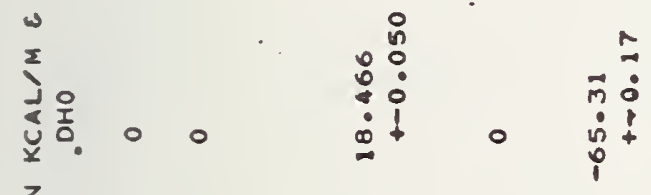

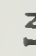

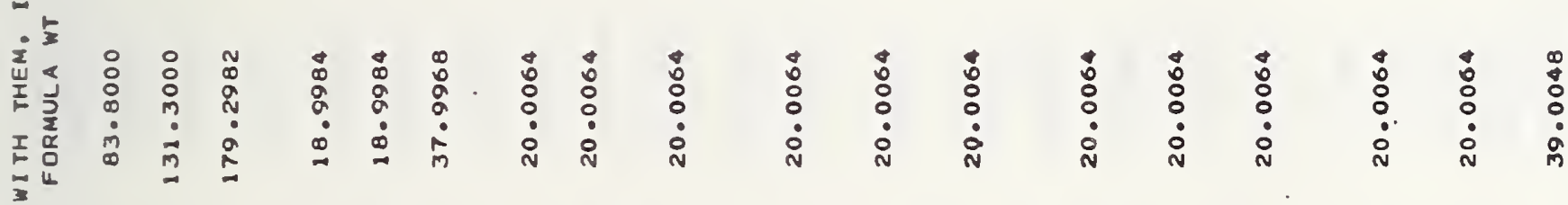

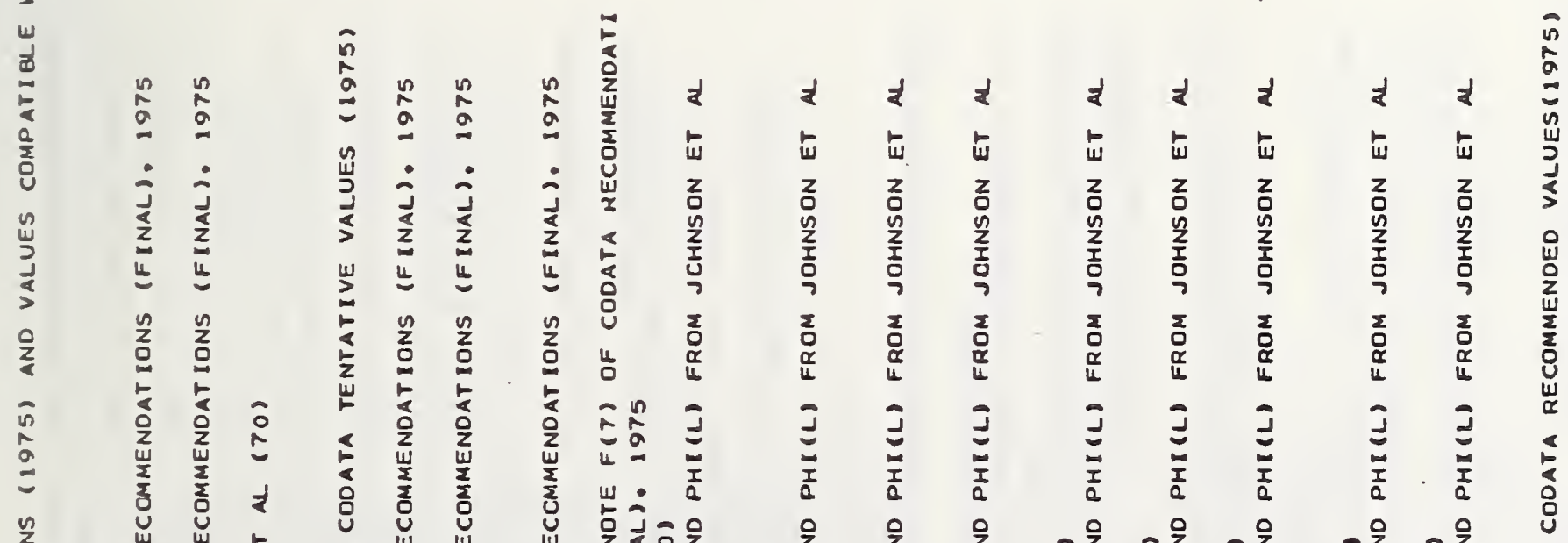

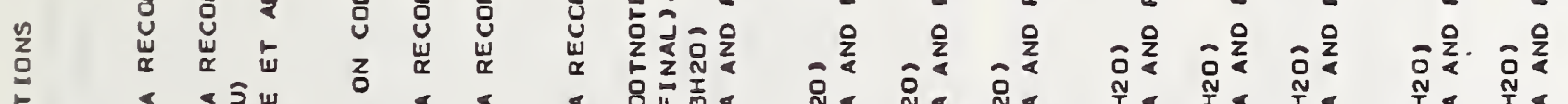

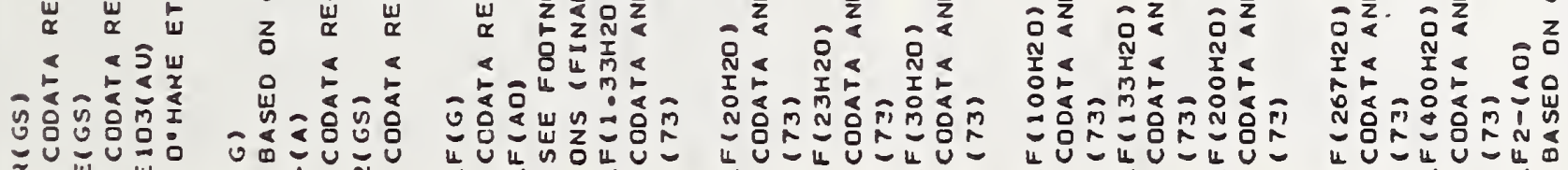

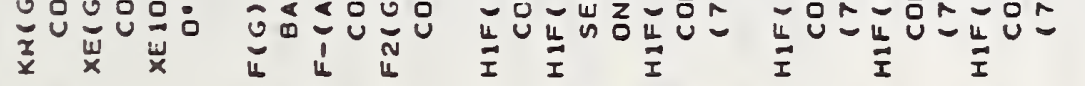

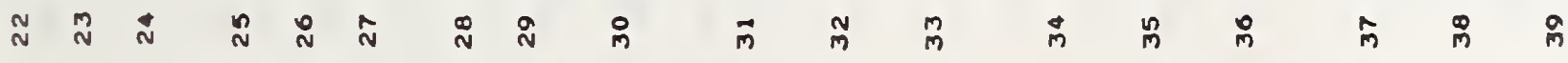




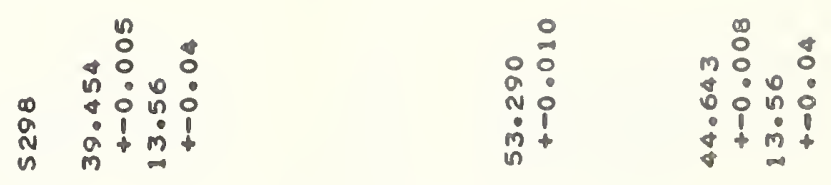

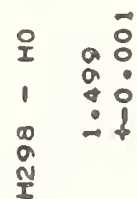

ํํㅇำ

ํ.

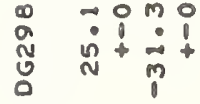

की

$\begin{array}{lll}N & \\ 0 & 1 & 0 \\ 0 & 0 & 0 \\ 0 & 0 & 0\end{array}$

ำ

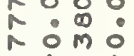

$\sim 101$

$N+\vec{m}$

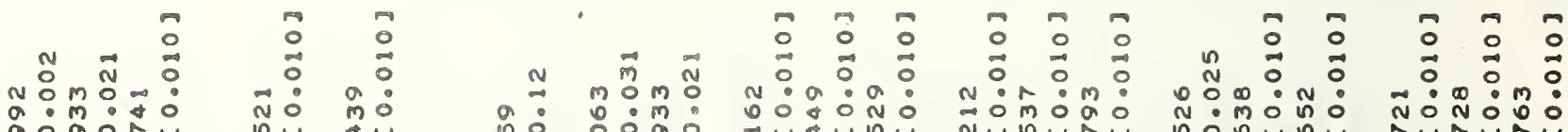

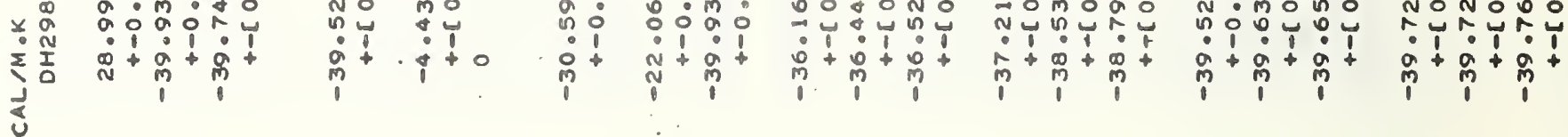

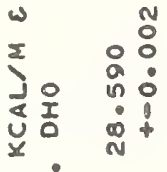

$z$

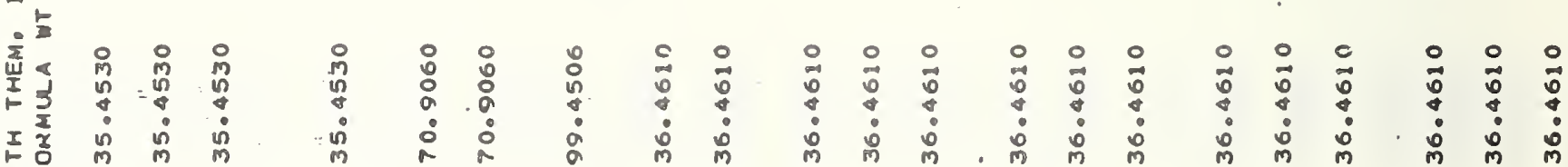

$\begin{array}{lllllllll} & & & & \\ 0\end{array}$


$\stackrel{\infty}{a}$

$\stackrel{0}{I}$
1
$\infty$
0
$\stackrel{N}{I}$

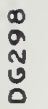

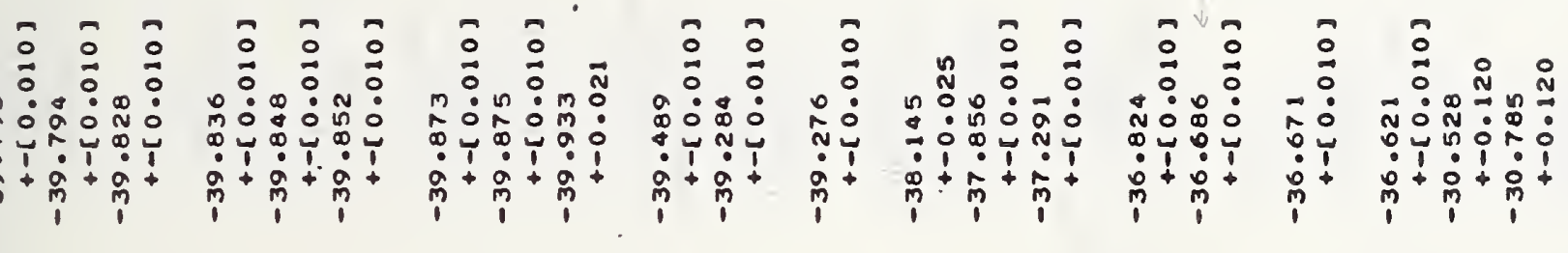
U

$\omega$

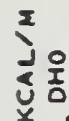

$z$

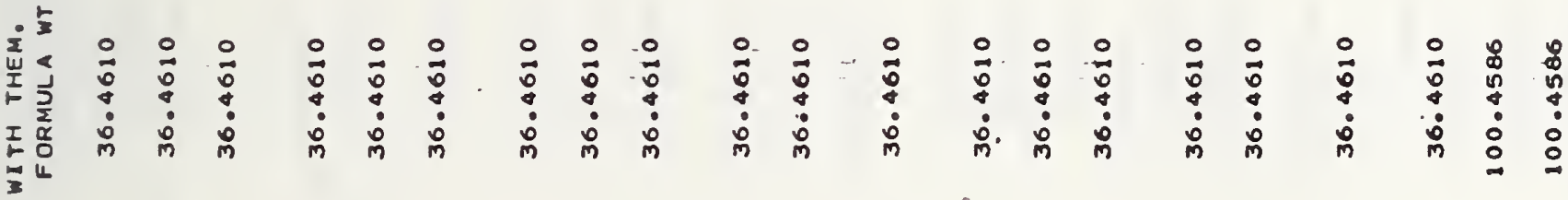

Ш

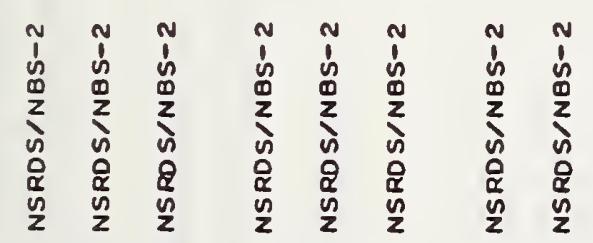

$$
\begin{aligned}
& \begin{array}{lll}
z & z & z \\
0 & 0 & 0 \\
\alpha & \alpha & z \\
4 & 4 & 4
\end{array}
\end{aligned}
$$

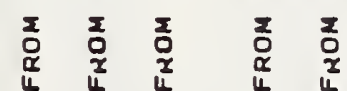

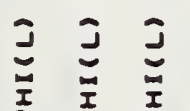

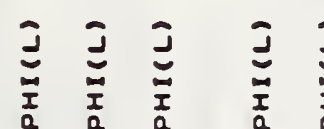

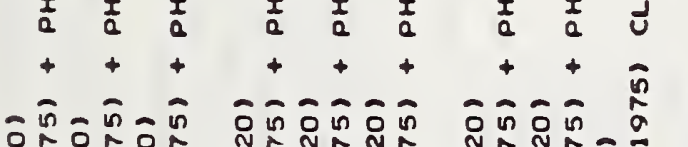

oำ

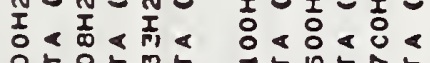

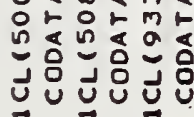

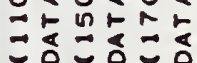

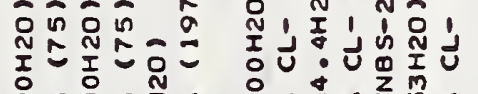

잔ㄷㄴ

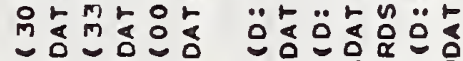

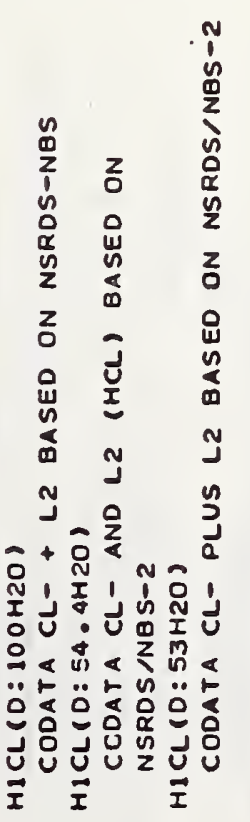

$\vec{I} \stackrel{\vec{I}}{I}$

İU Ư

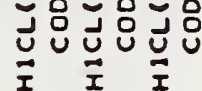

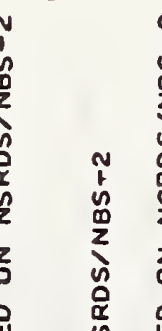

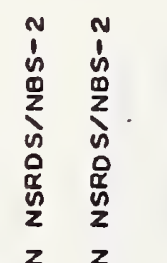

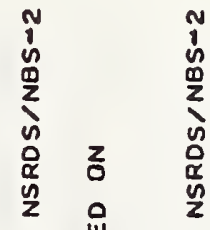

$\begin{array}{ll}n & n \\ 1 & 1 \\ 0 & 0 \\ 0 & 0 \\ 2 & 2 \\ 0 & \text { n } \\ 0 & 0 \\ 0 & 0 \\ 0 & n\end{array}$

० ก

$:$ : :

ก

$\therefore=\pi$ 
ำ 용

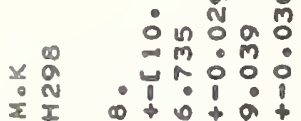

\section{Cor}

3

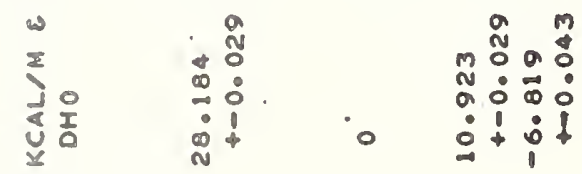
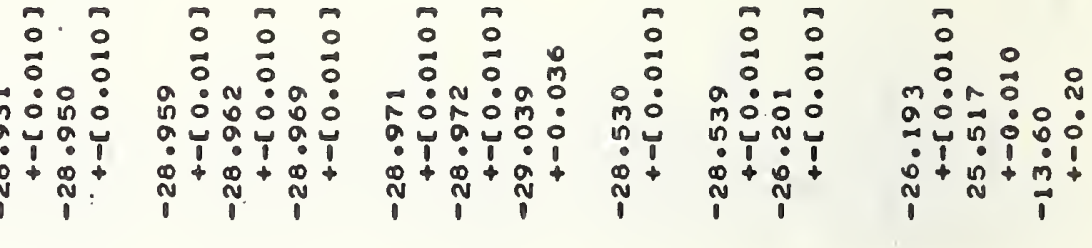

3

\section{$+$}

\section{1}

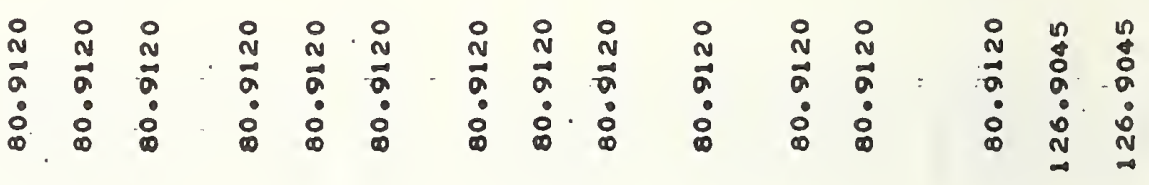
E 


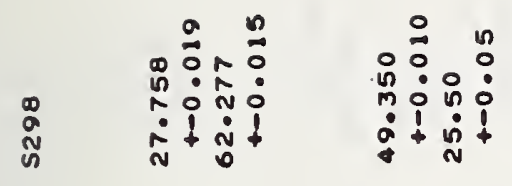

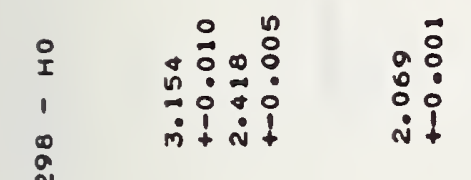

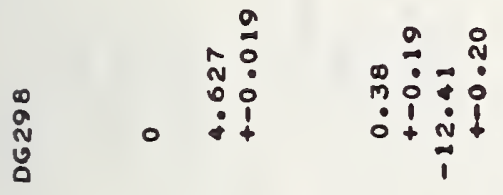

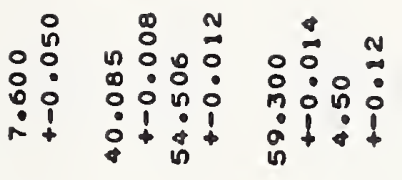

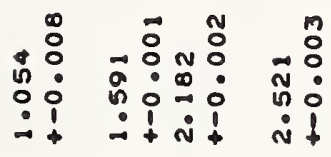
- 5 a

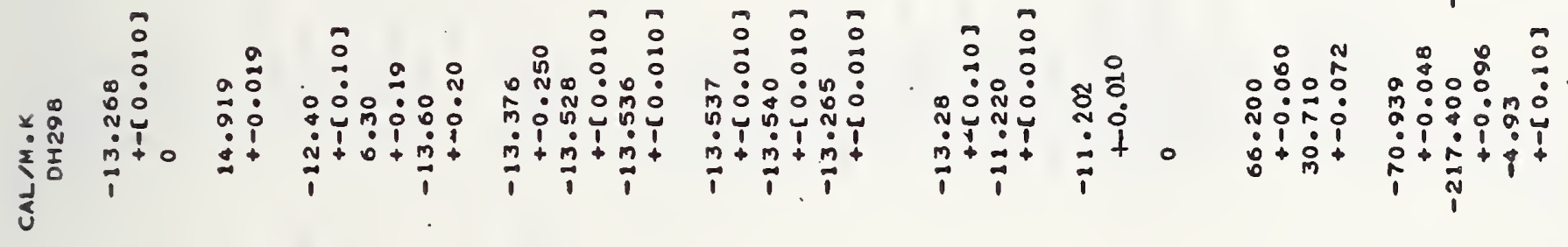

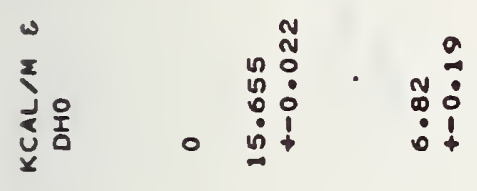

$z$

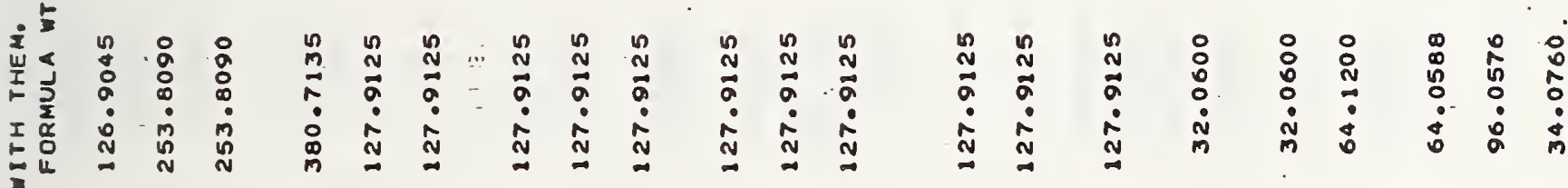

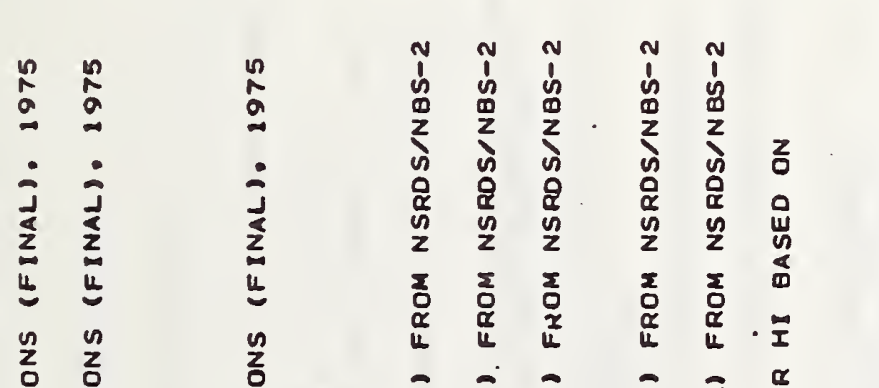

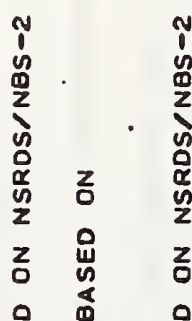

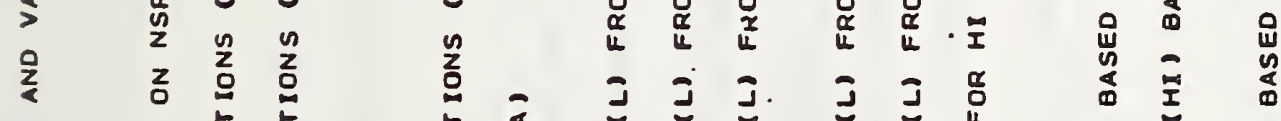

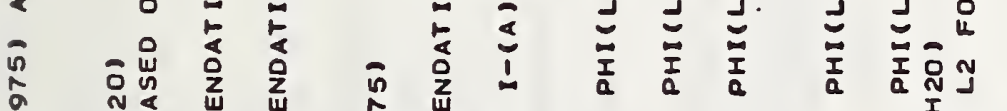

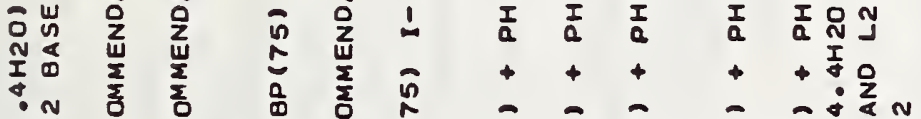

$\therefore$ J

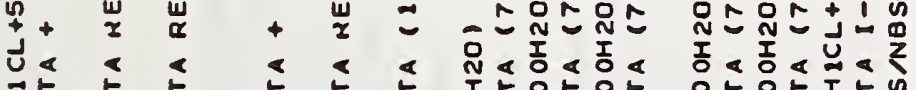

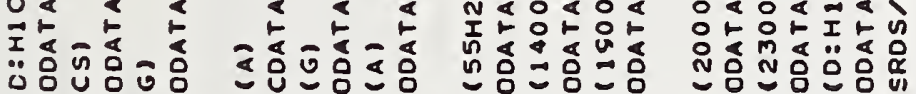

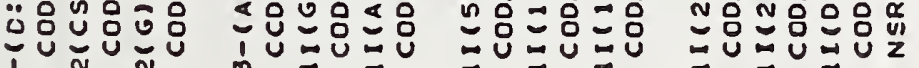

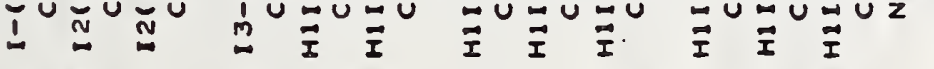

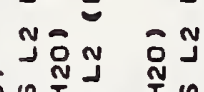

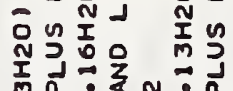

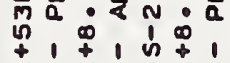

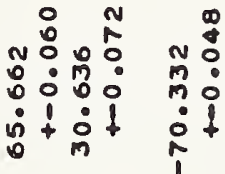

U.

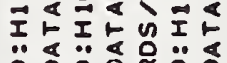

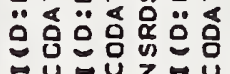

$\vec{I} \underset{I}{\vec{I}}$

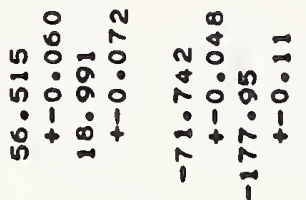

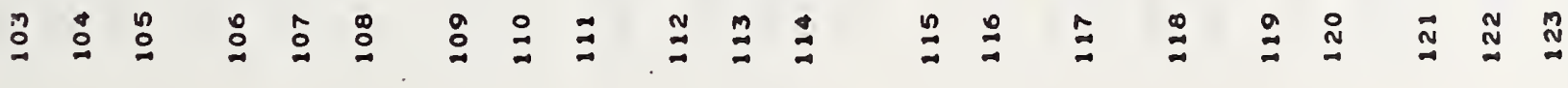


$\begin{array}{lll} & & 0 \\ & \infty & \vdots \\ \infty & 0 & 0 \\ 0 & 0 & 1 \\ N & 0 \\ N & N\end{array}$

0
$I$
1
0
0
$N$
$I$

ำ

$\begin{array}{ll}N & 0 \\ 0 & 0\end{array}$

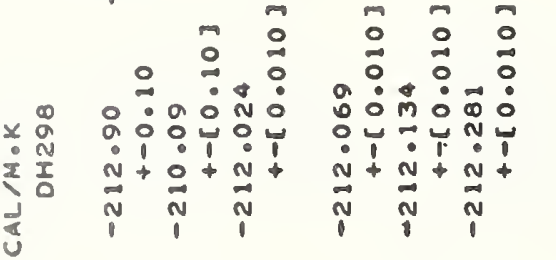

$\sum_{\substack{2 \\ x}}^{\infty}$

$z$

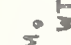

\section{$\begin{array}{llllll}0 & 0 & 0 & 0 & 0 & 0 \\ 0 & M & M & M & M & M \\ 0 & 0 & 0 & 0 & 0 & 0 \\ \stackrel{0}{0} & 0 & 0 & \dot{0} & \dot{0} & \dot{0}\end{array}$}
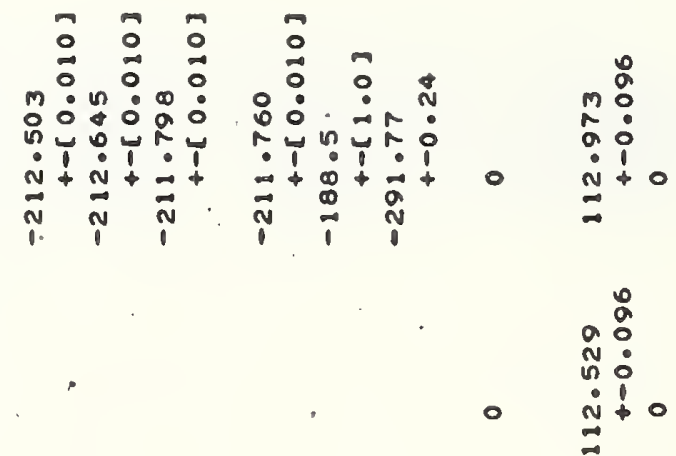

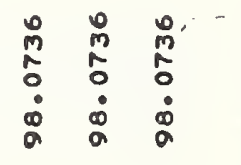

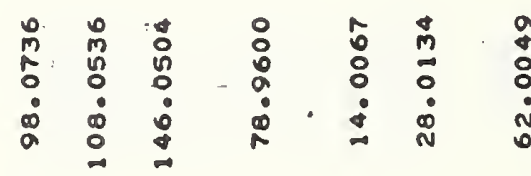

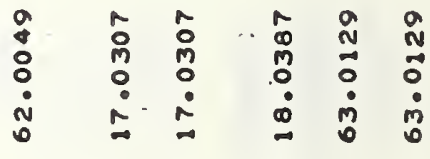

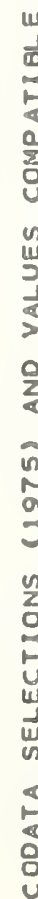

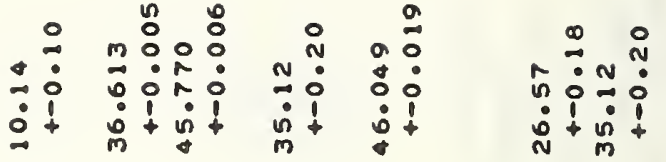

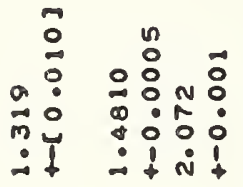

$\begin{aligned} & \text { : } \\ 0 & : \\ \dot{0} & \dot{0}\end{aligned}$

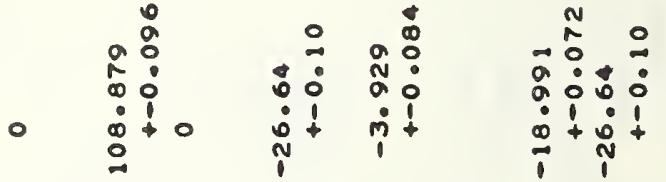




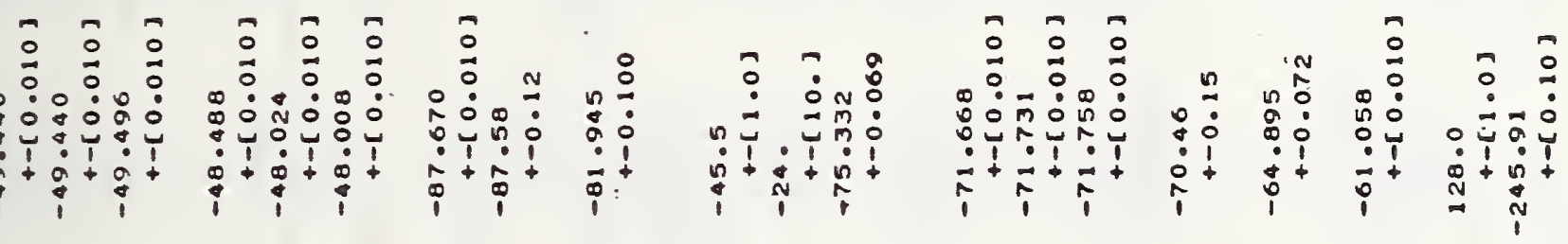

운

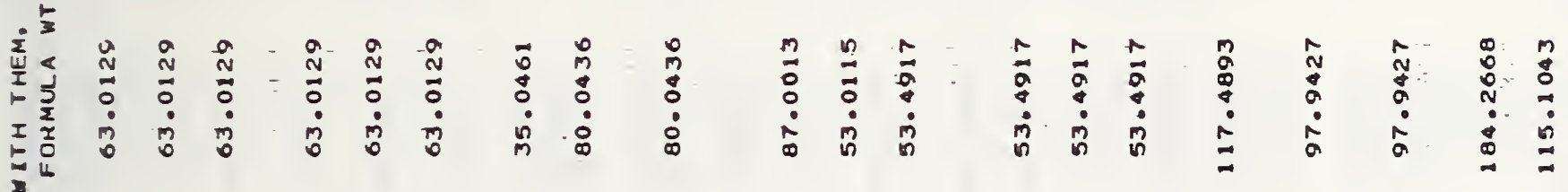




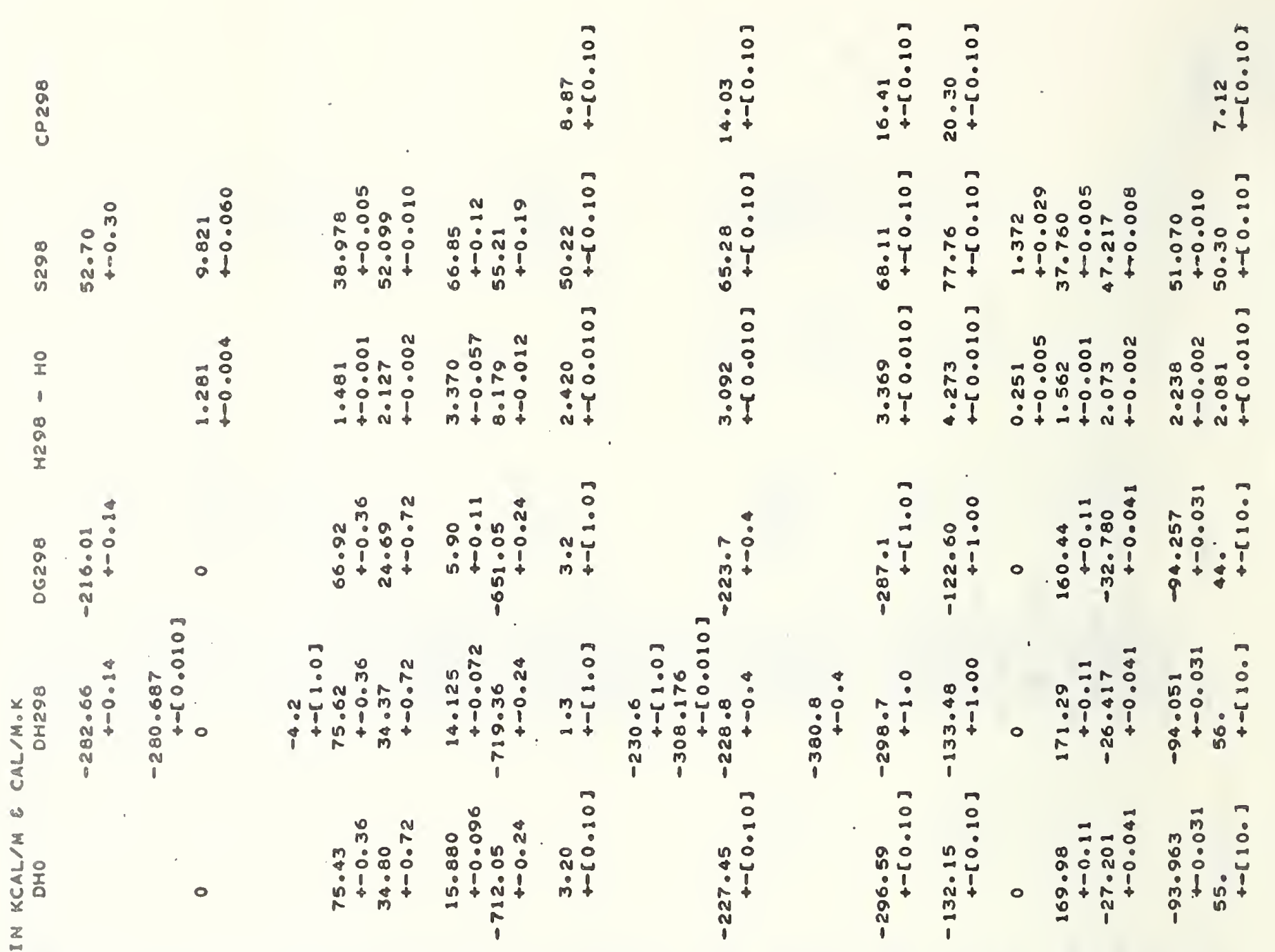

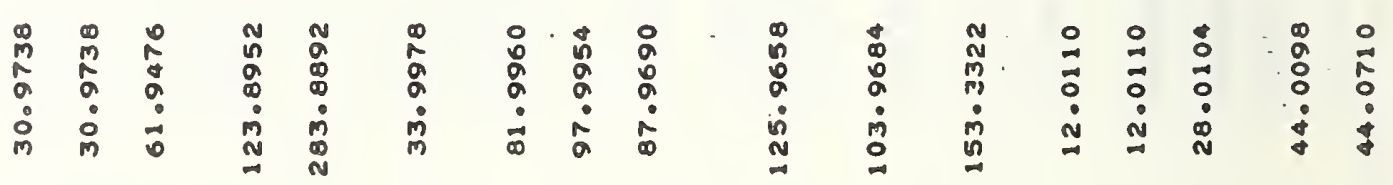

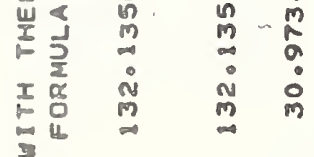
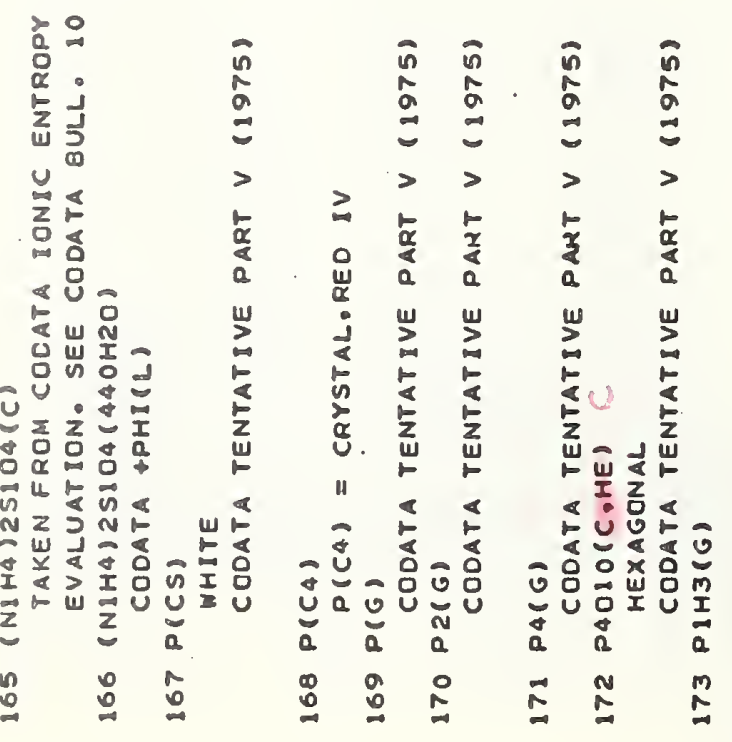


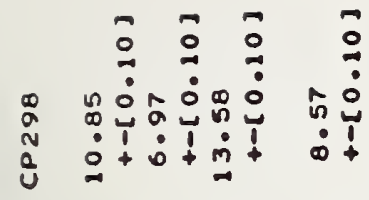

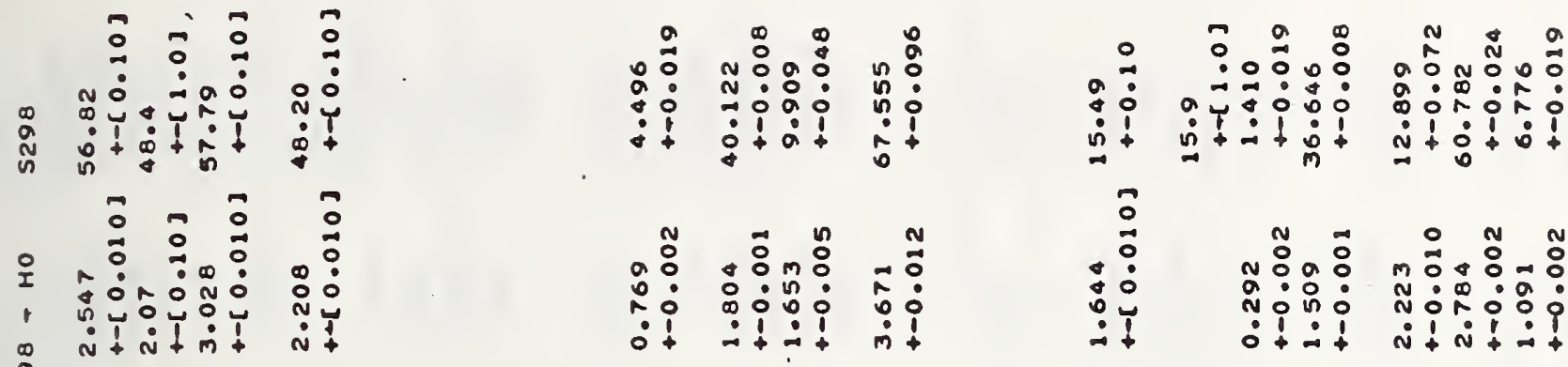

\%

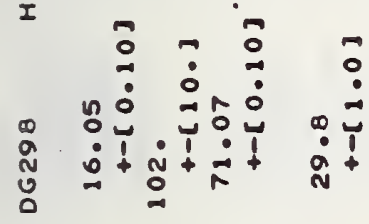

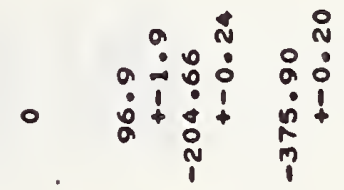

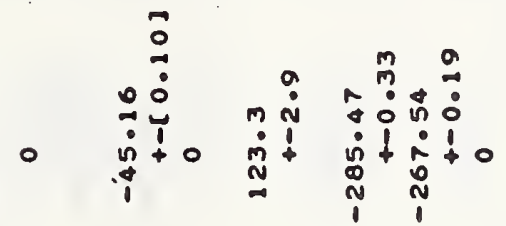

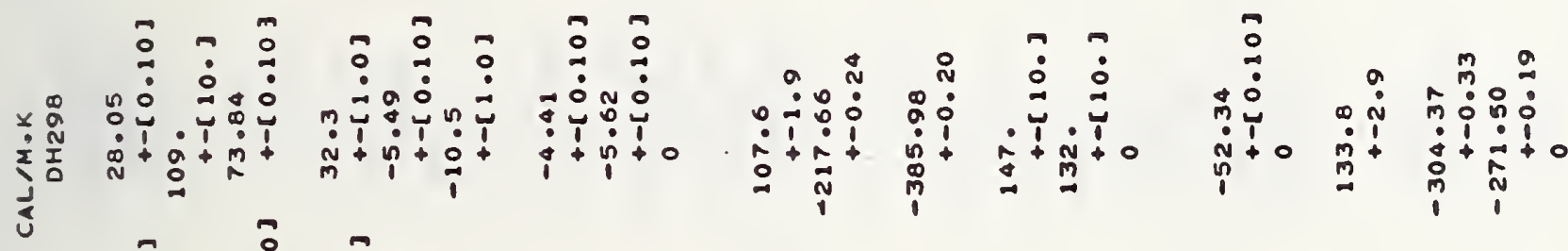

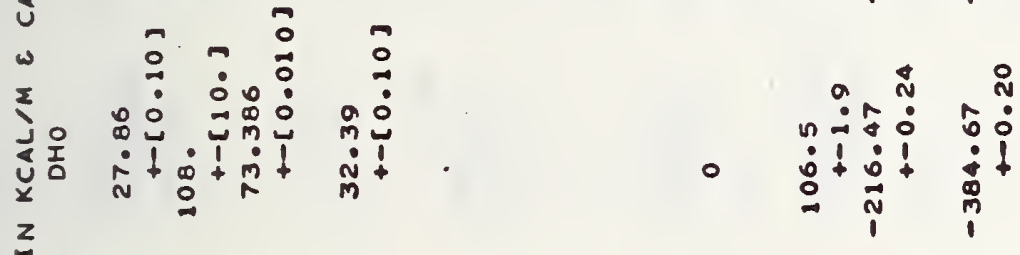

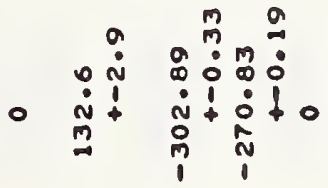

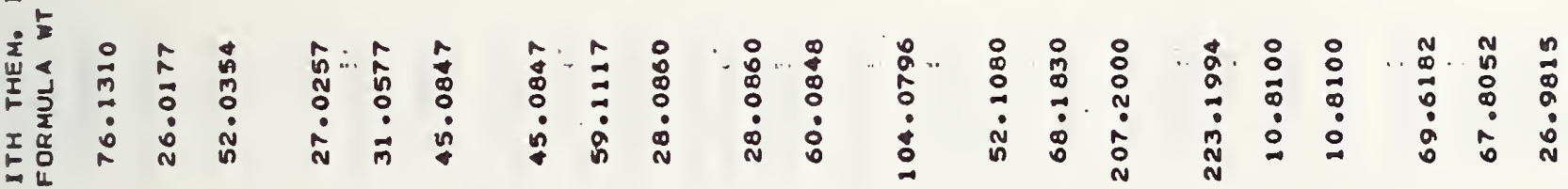

2

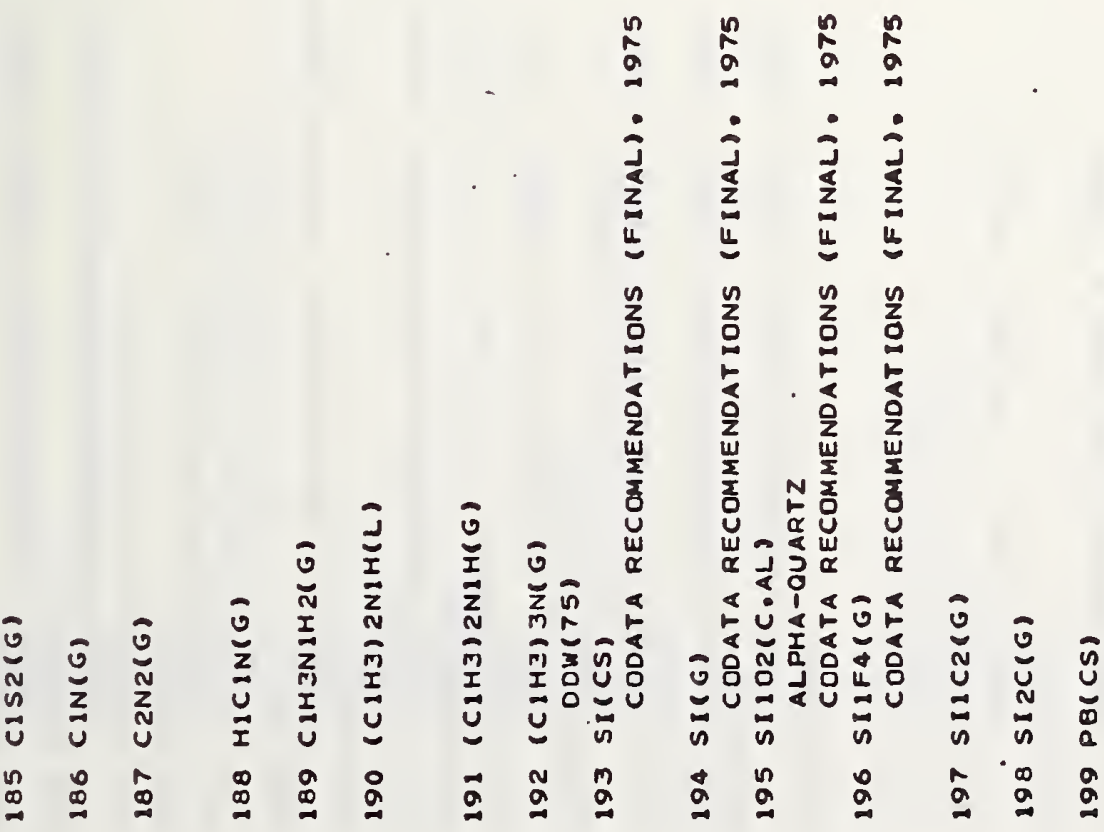

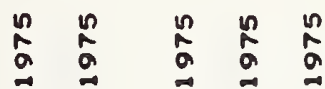

药

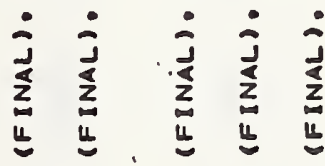

난

a

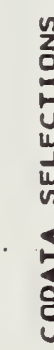

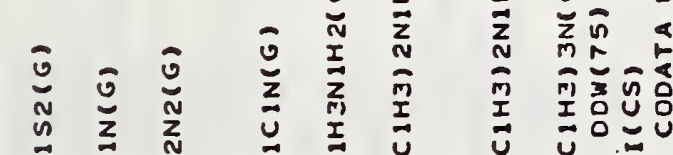

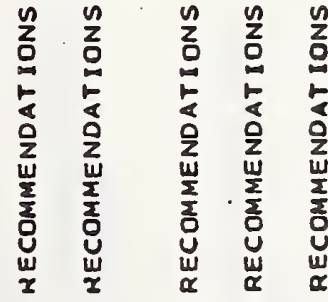

\&

吊

$\stackrel{2}{a}$ 


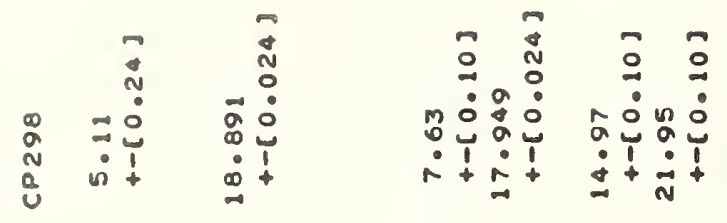

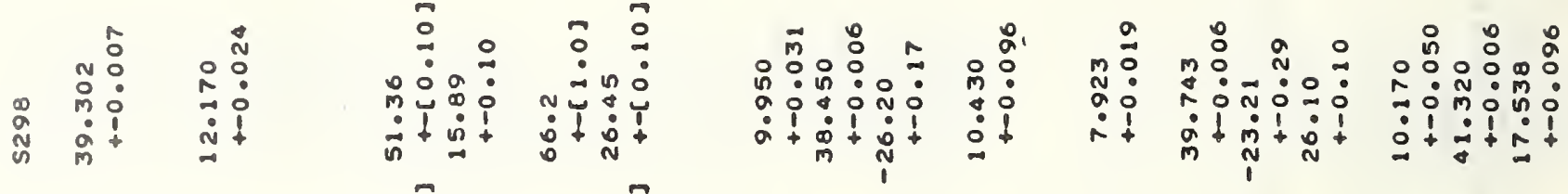

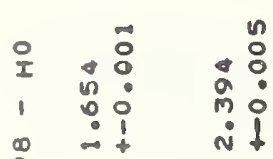

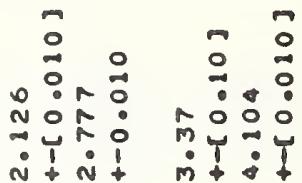

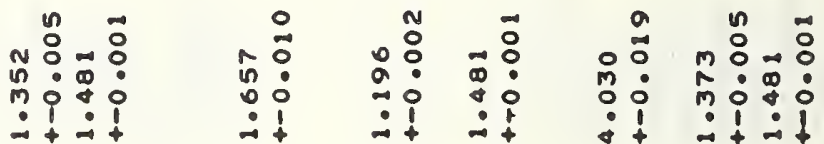

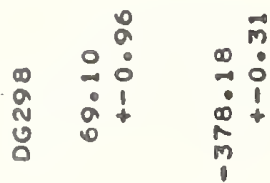

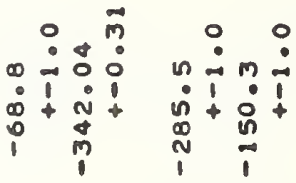

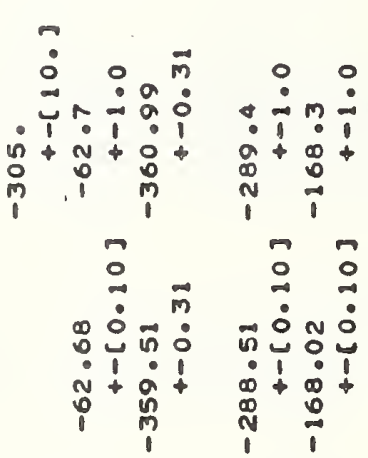

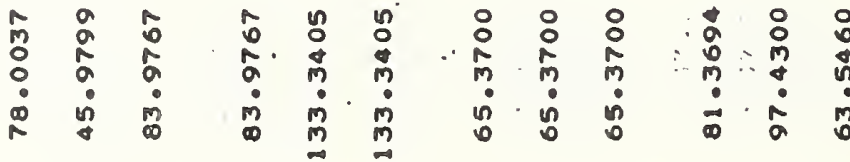

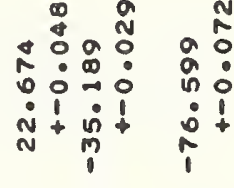

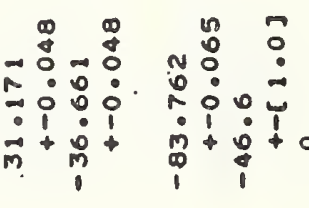

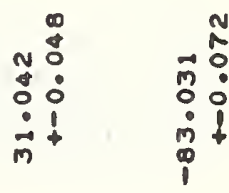

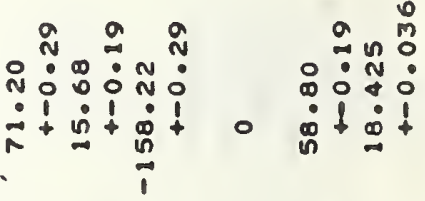

ก 2 a 2 ก ก ํํํํํำ i:i i o i i i

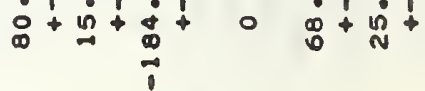

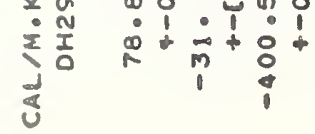

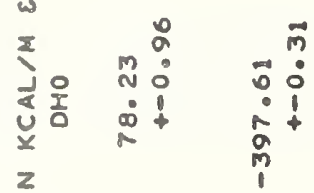

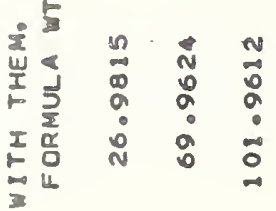

足证

ล $\frac{2}{2}$

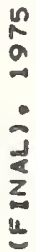

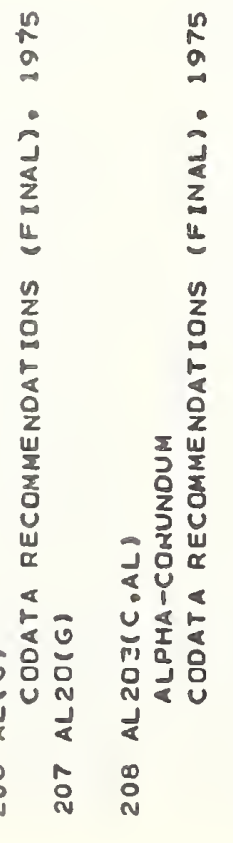

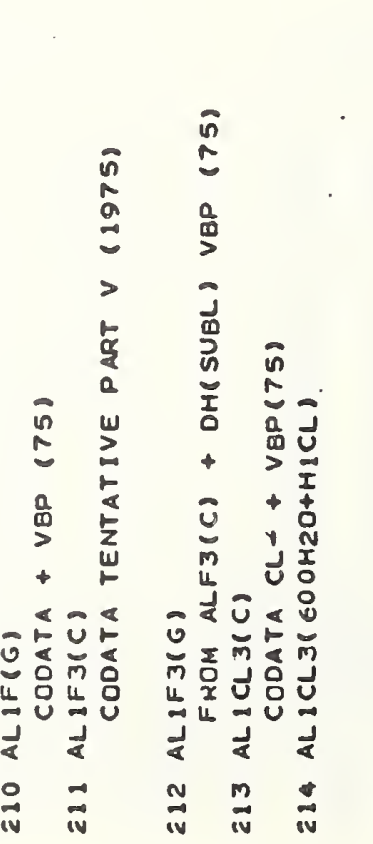

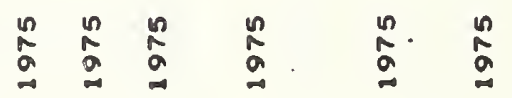

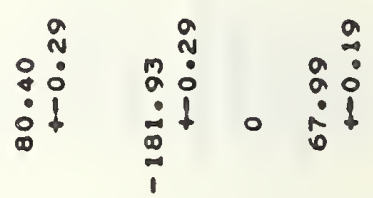

$\begin{array}{llllll}\circ & 0 & 0 & 0 & 0 & 0 \\ 0 & 0 & 0 & 0 & 0 & 0 \\ 0 & 0 & 0 & 0 & 0 & 0 \\ 10 & 0 & 0 & 0 & 0 \\ \dot{0} & 0 & 0 & 0 & 0 & 0 \\ 0 & 0 & 0 & 0 & 0 & 0 \\ 0 & 0 & 0 & 0\end{array}$

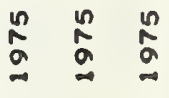

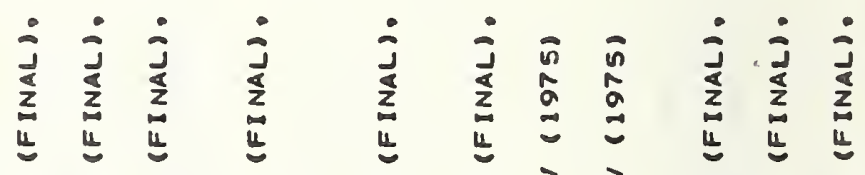

$\begin{array}{lll}n & n & n \\ 0 & 0 & 2 \\ 0 & 0 & 0\end{array}$

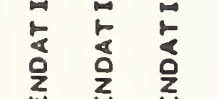

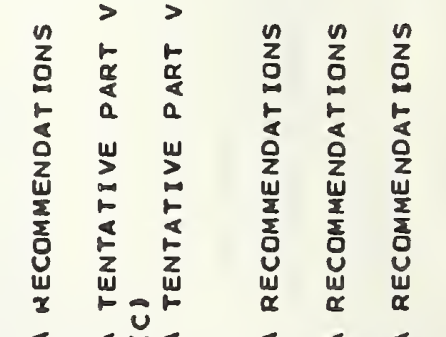

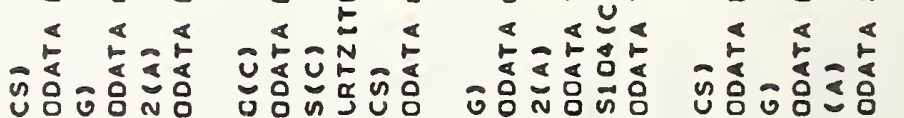

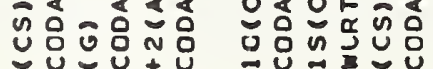

$\sum_{N} \sum_{N} \sum_{N} \sum_{N} \sum_{N} \sum^{2}$

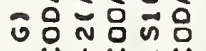

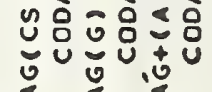

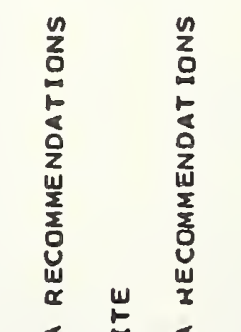

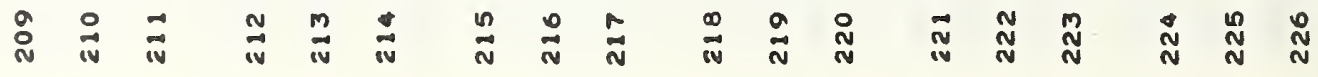




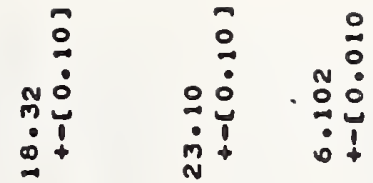

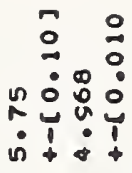

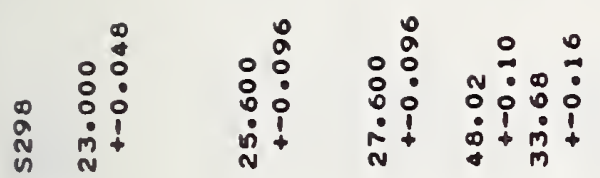

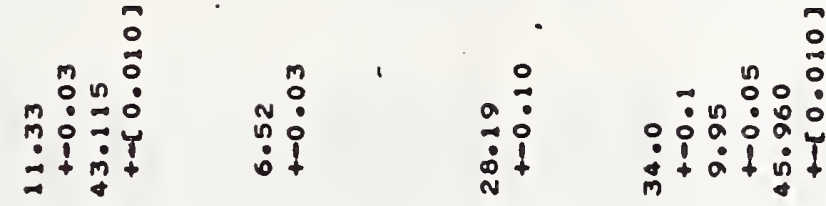

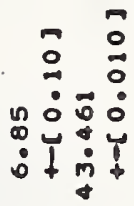

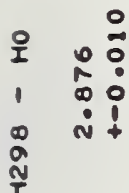

i⿱

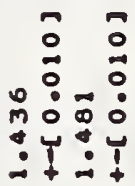

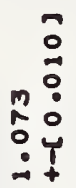

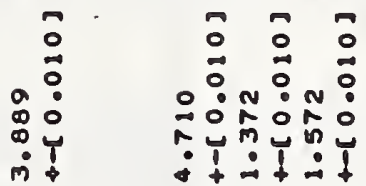

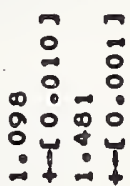

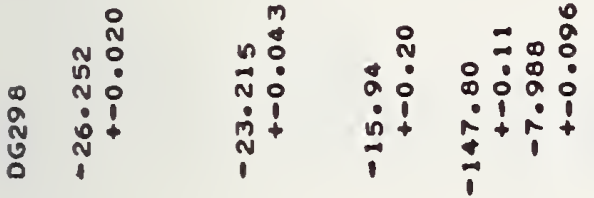

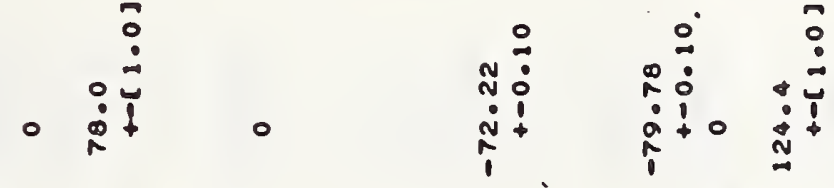

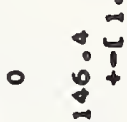

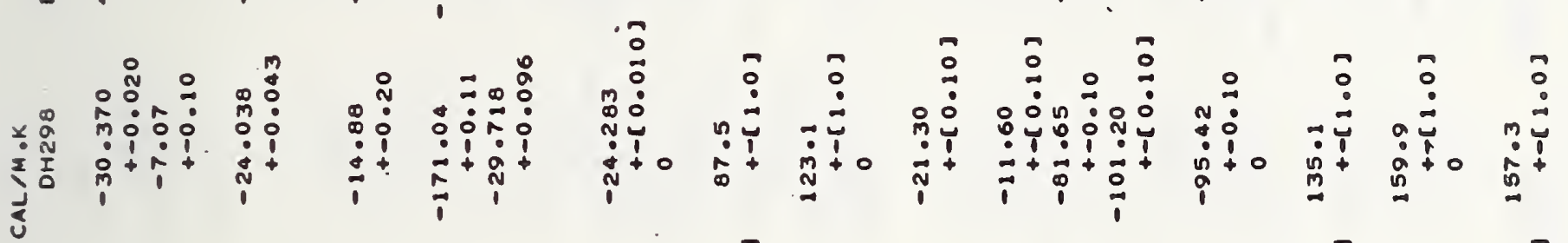

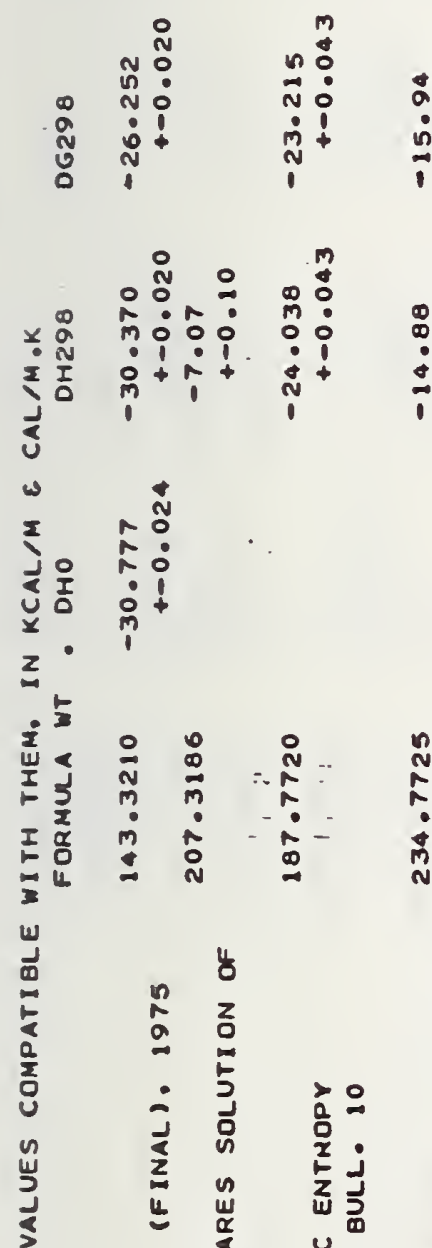

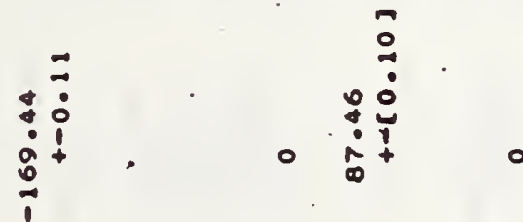

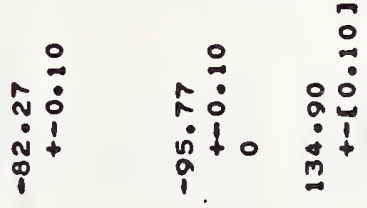

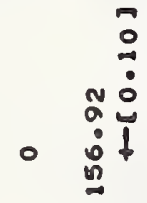

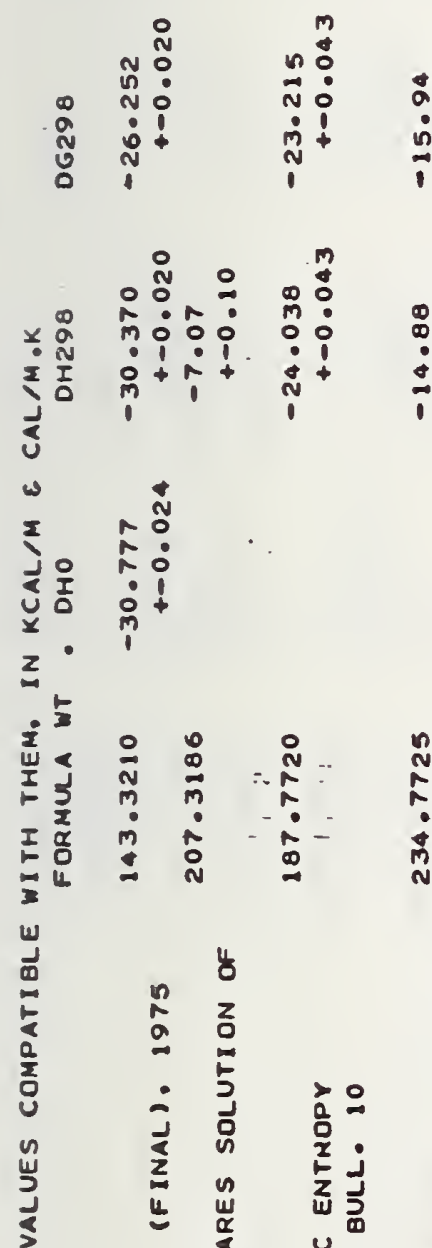

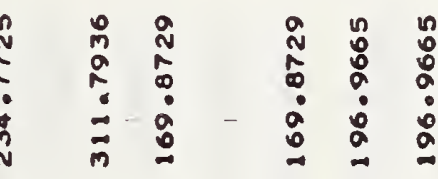

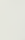

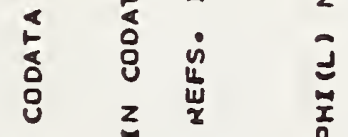

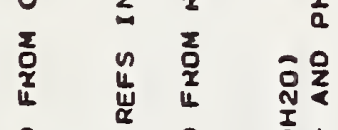

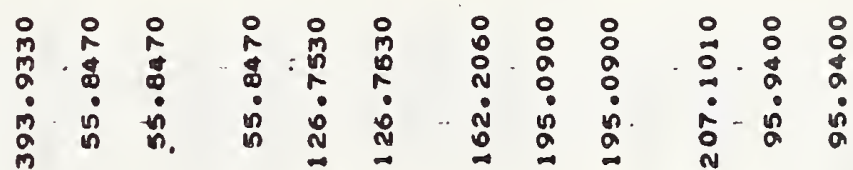

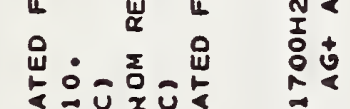

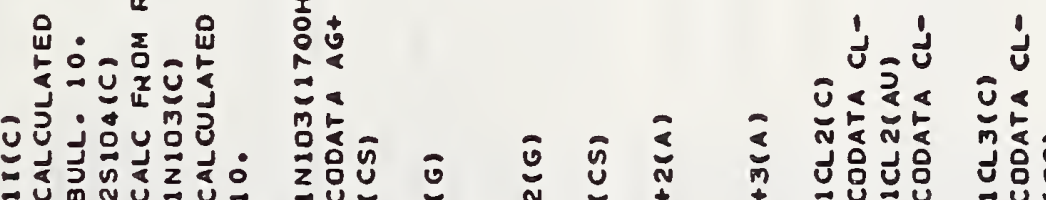

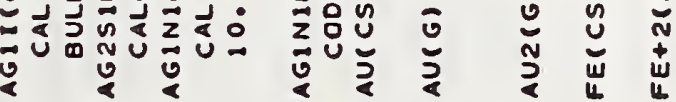

$\stackrel{N}{N} \stackrel{N}{N}$
ฉ

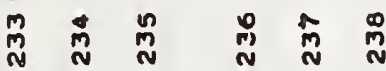

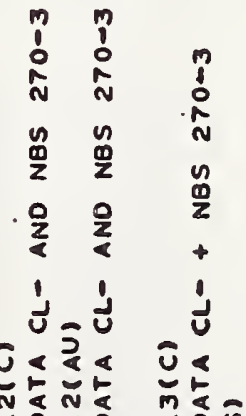

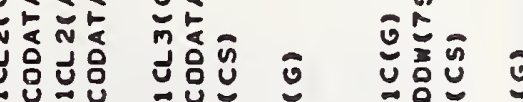
岁诺

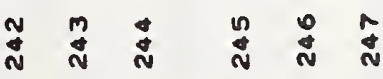




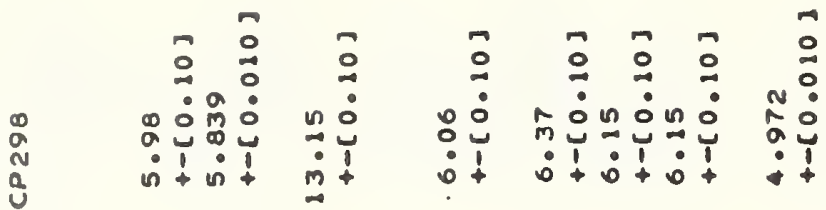

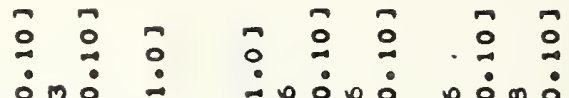
的色足n

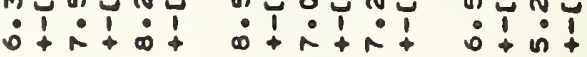
$+N+\infty+\infty+N+N+\infty$

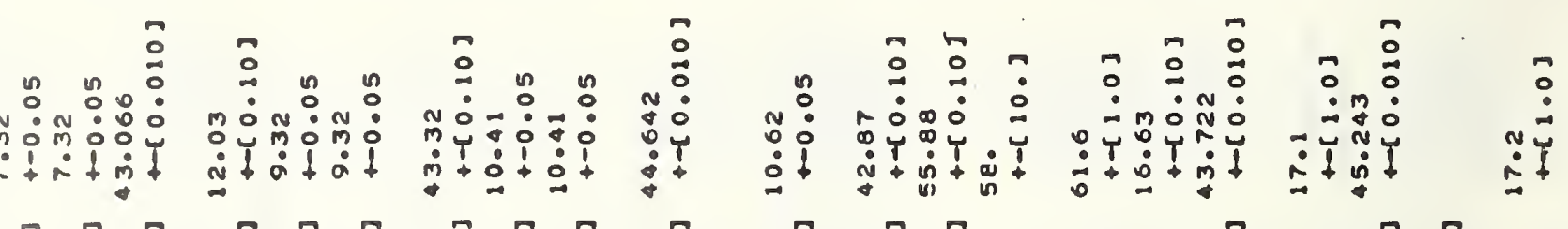

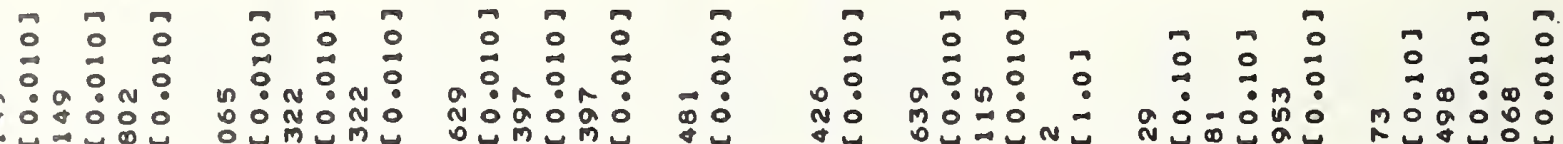

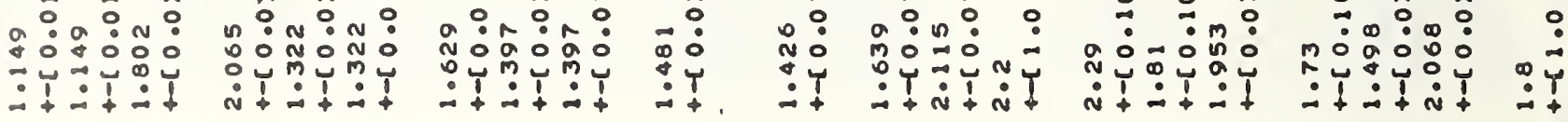

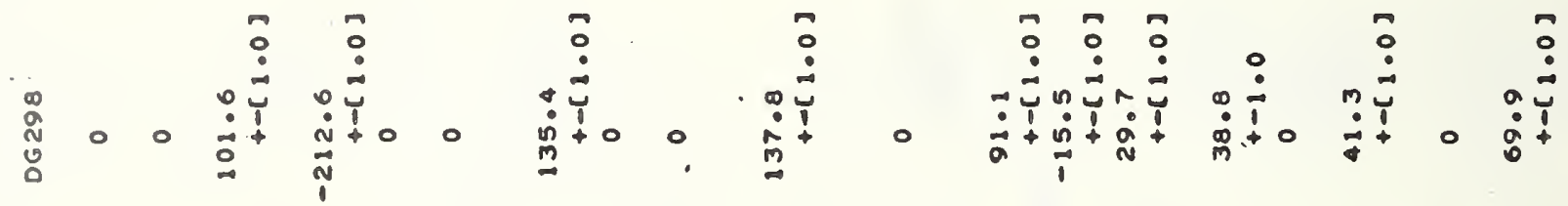

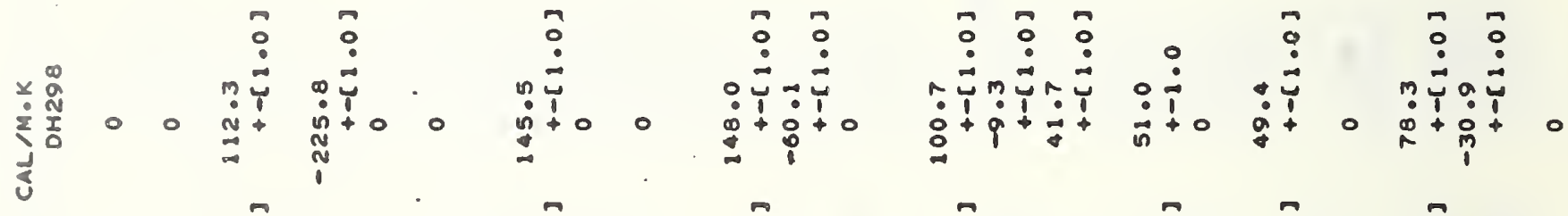

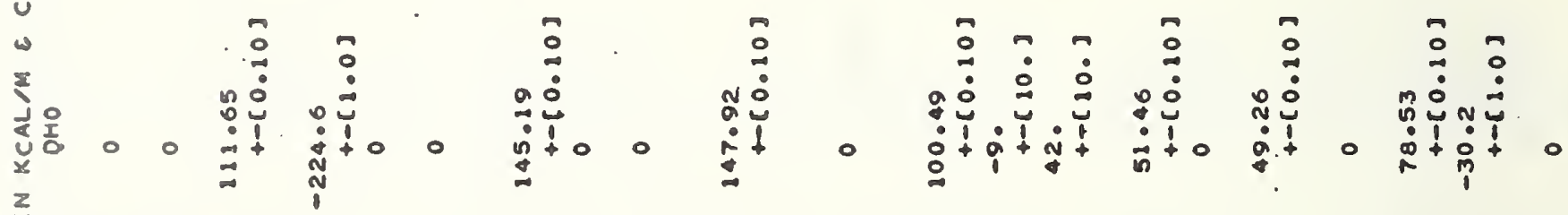
2

 


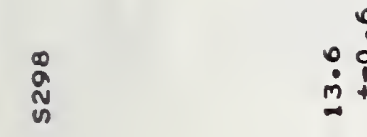

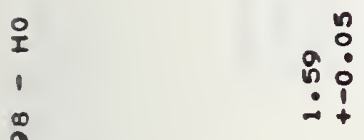

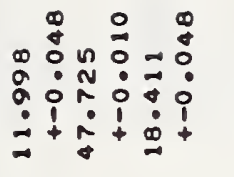

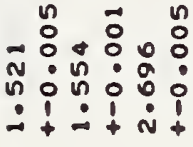

$\stackrel{0}{0}: \stackrel{0}{0}:$

$\stackrel{\dot{m}}{\dot{m}}+\stackrel{i}{i} \stackrel{i}{N}+$

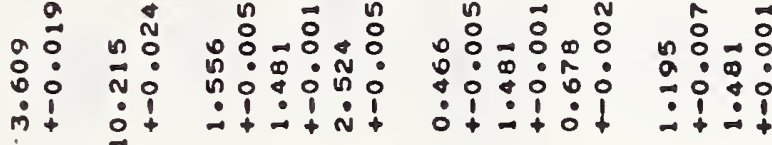

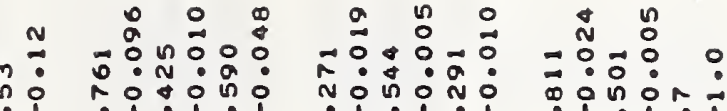

í

?.

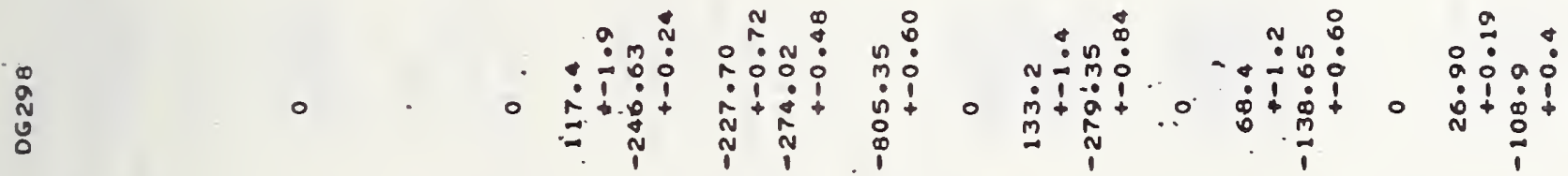

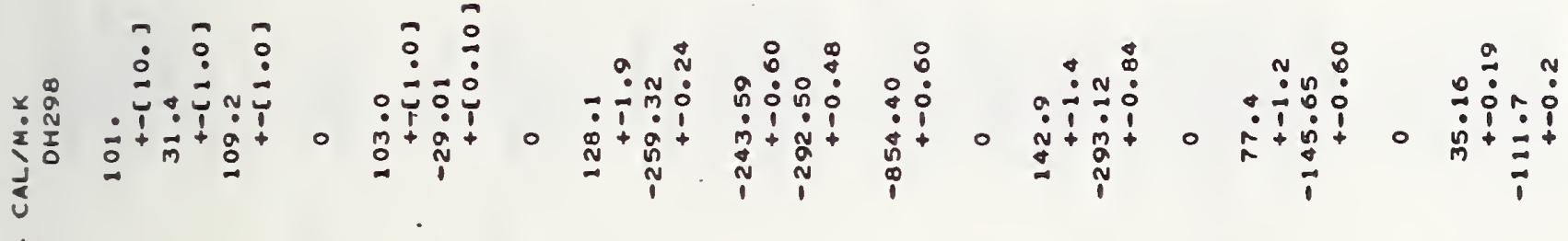

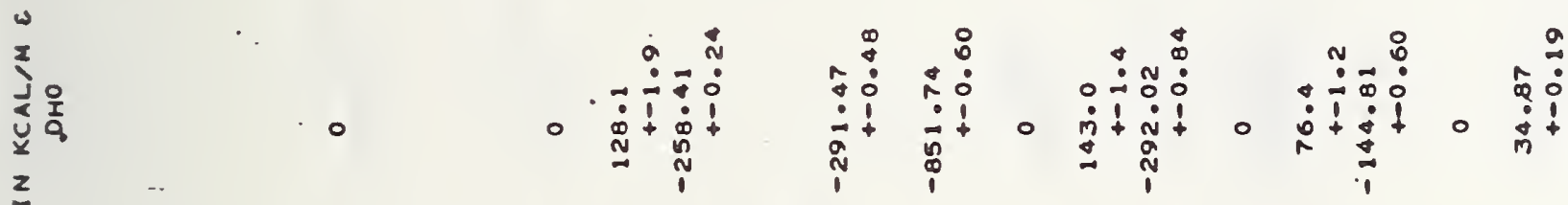

管

垔

mi 


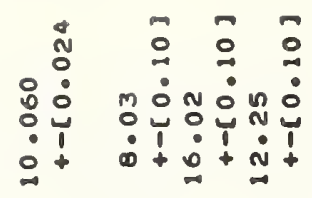

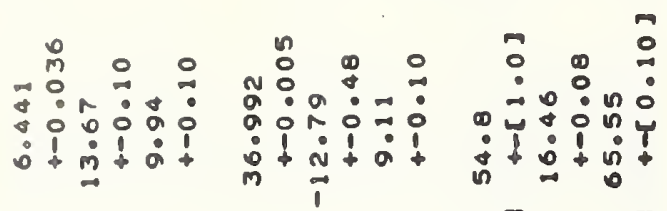

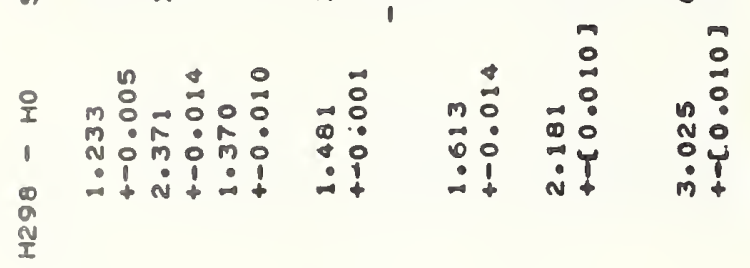

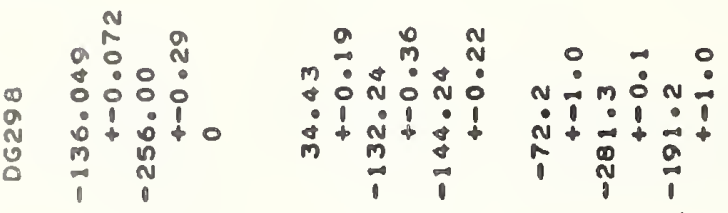

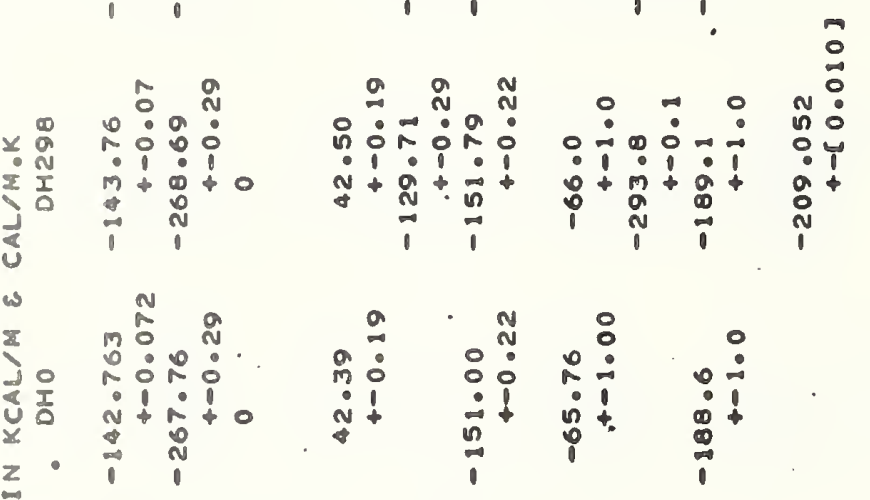

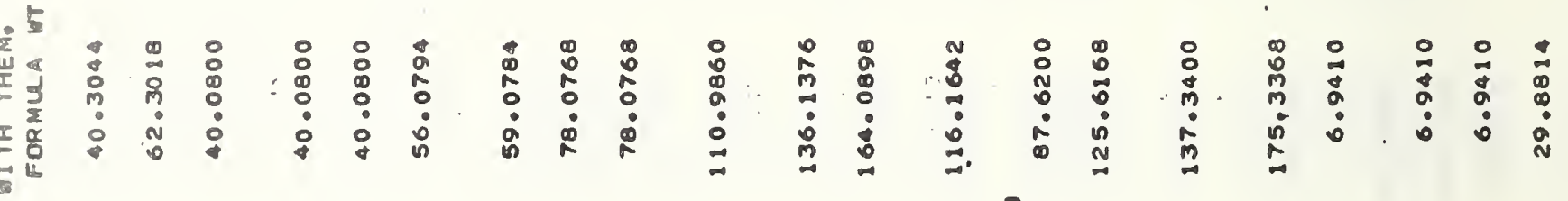

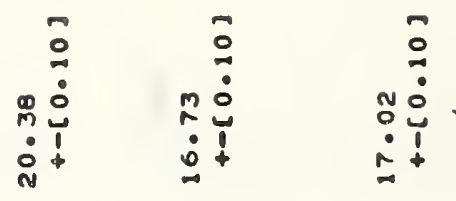

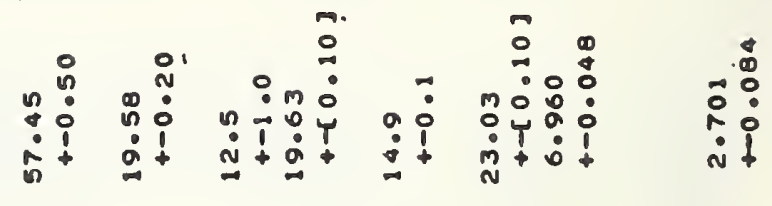

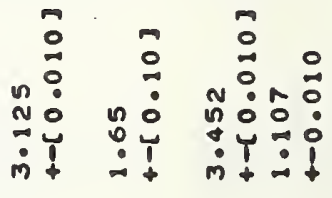

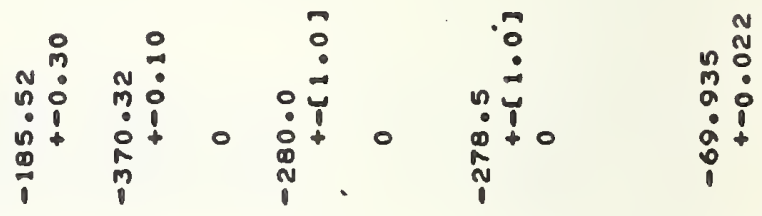

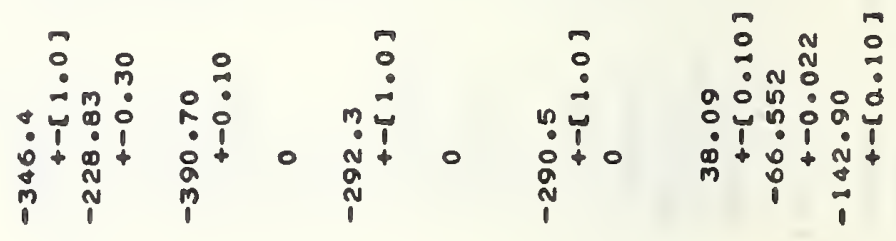

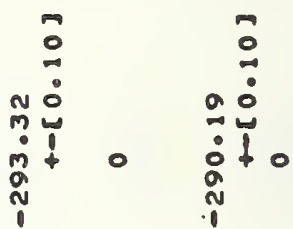

品

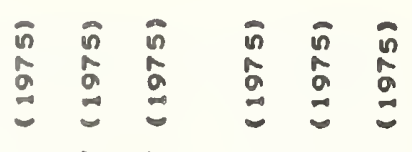

$>>>>>>$

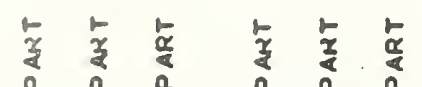

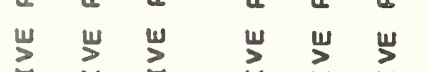

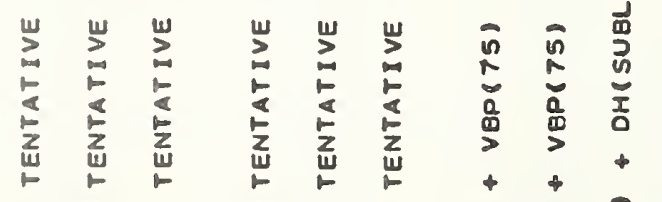

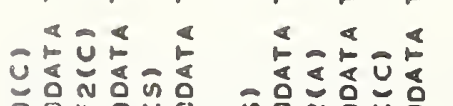

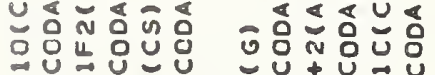

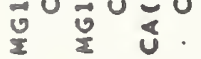

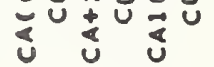

$+++$

过经过

U⿺辶寸

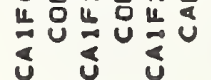

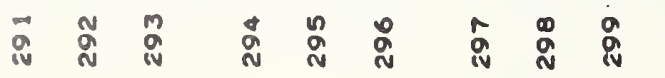

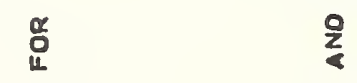

突

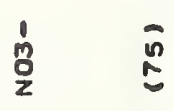

袋

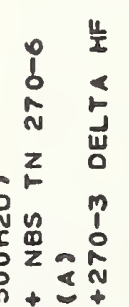

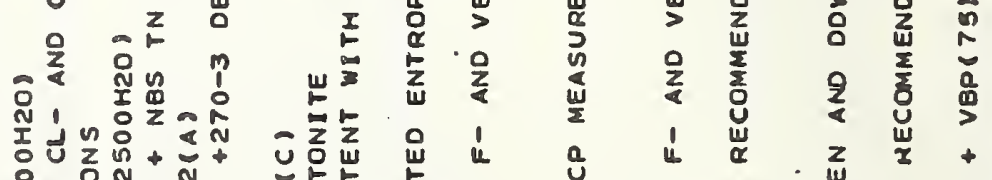

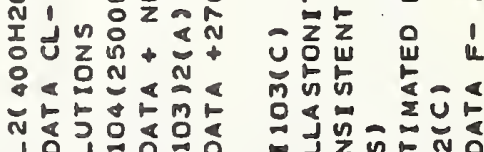

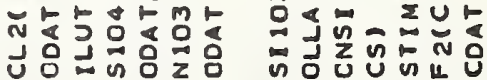

过吅过役

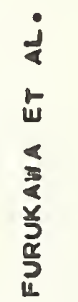

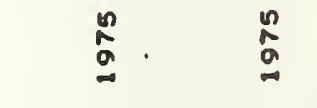

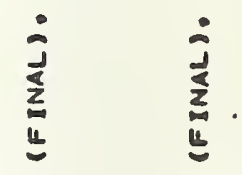

‡ $\sum_{0}^{n}$ ล $z_{0}^{n}$

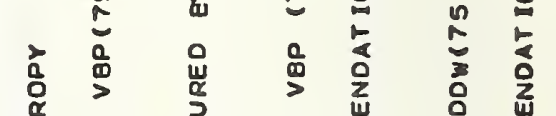

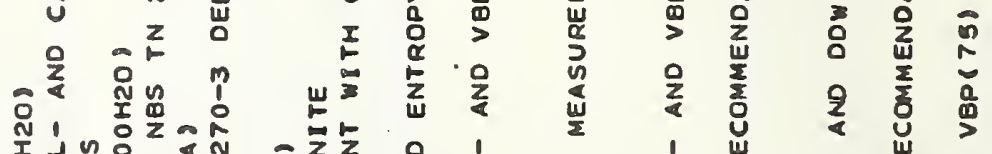

茄品品 


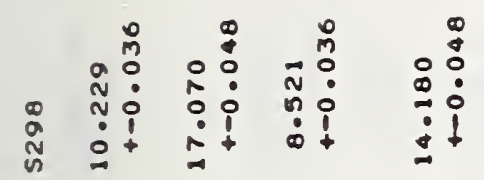

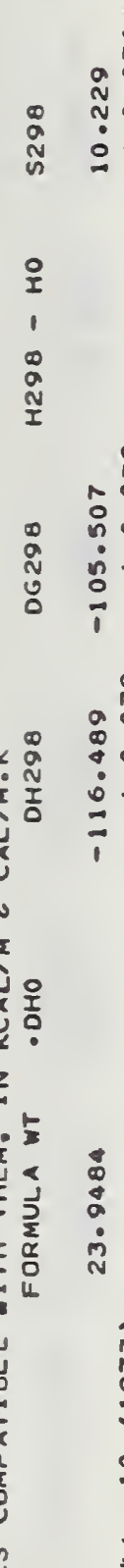

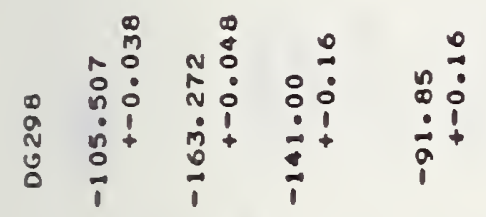

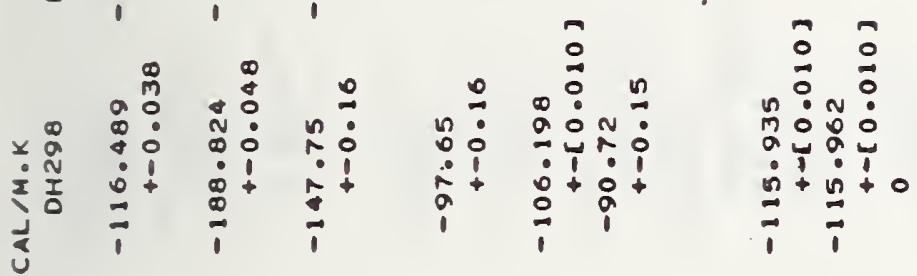

$\sum_{\substack{j \\ z}}^{\infty}$

z

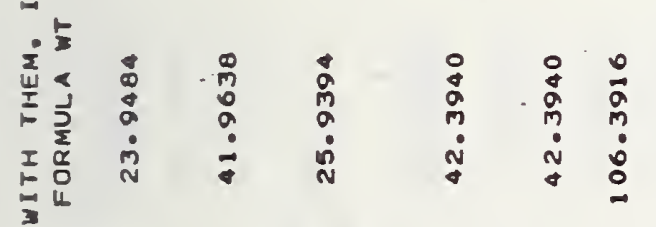

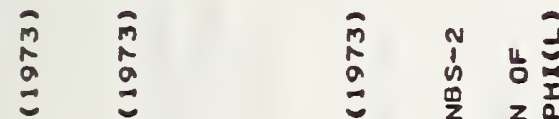

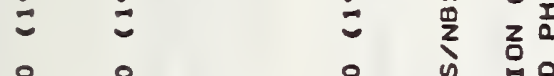

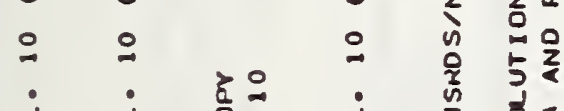

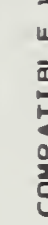

.

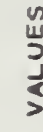

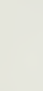

(1)
:

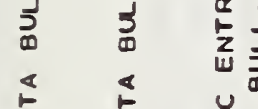

$\begin{array}{lll}3 & 0\end{array}$

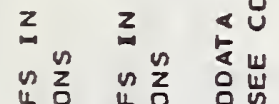

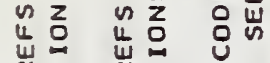

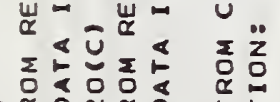

IUư

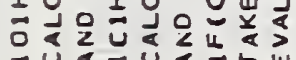

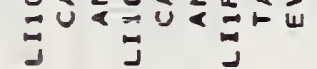

$\stackrel{m}{m} \stackrel{m}{m} \frac{n}{m} \stackrel{0}{m}$
\& $\quad$ \&

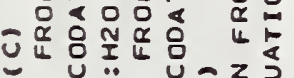

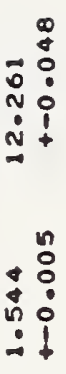

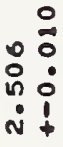

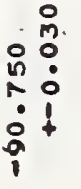



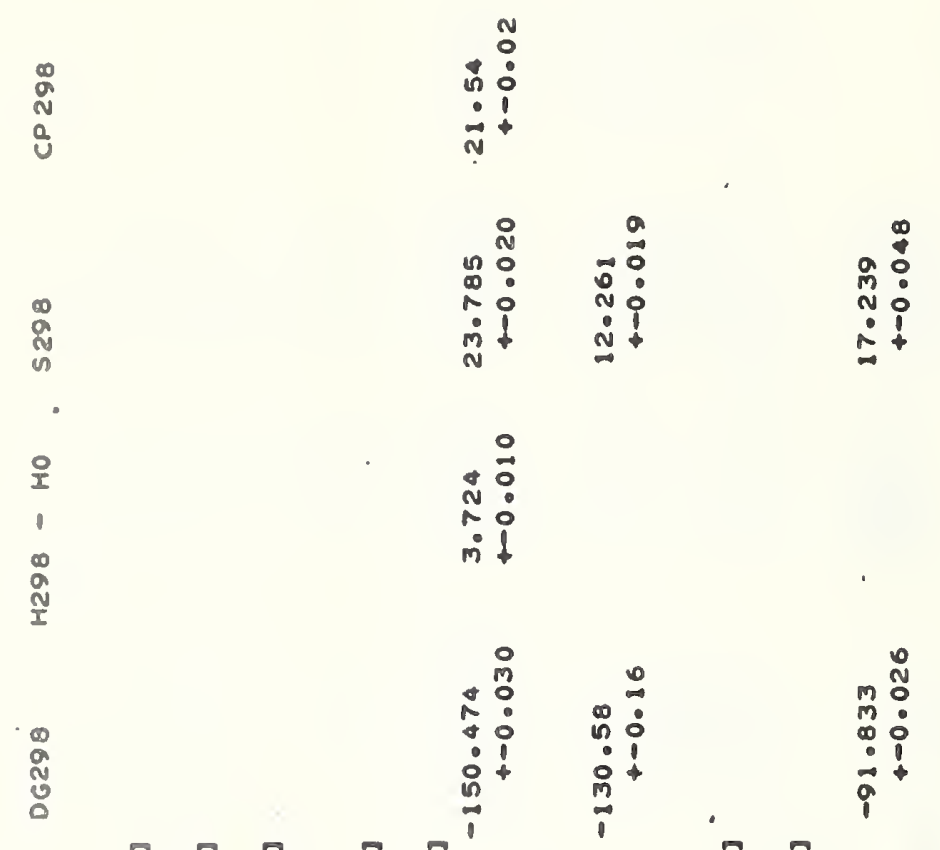

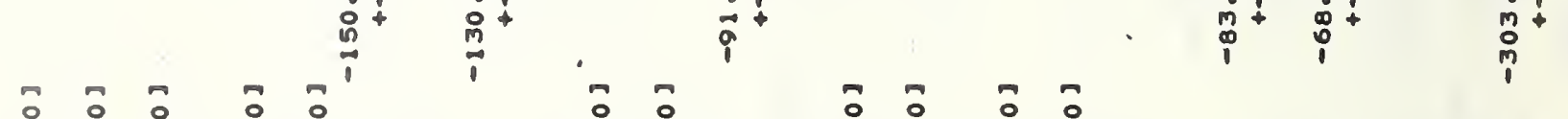

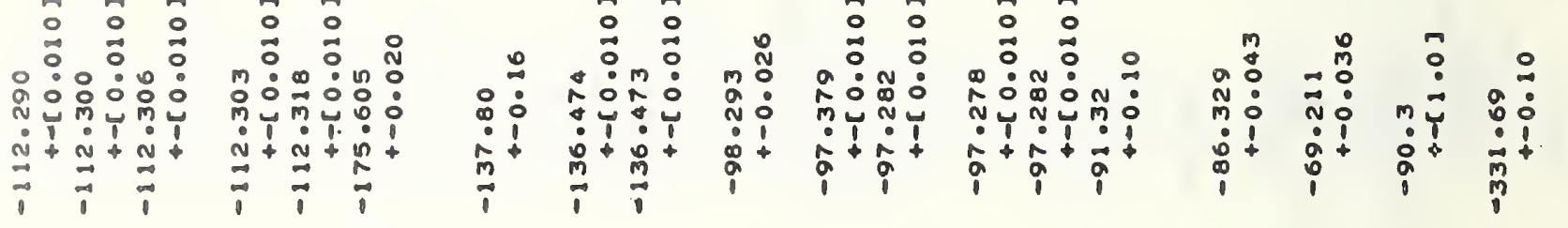

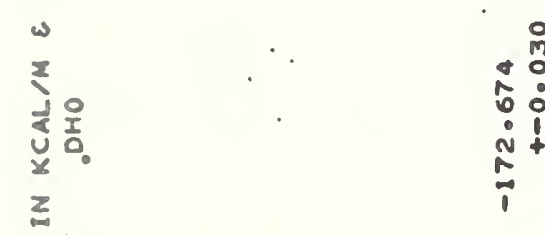
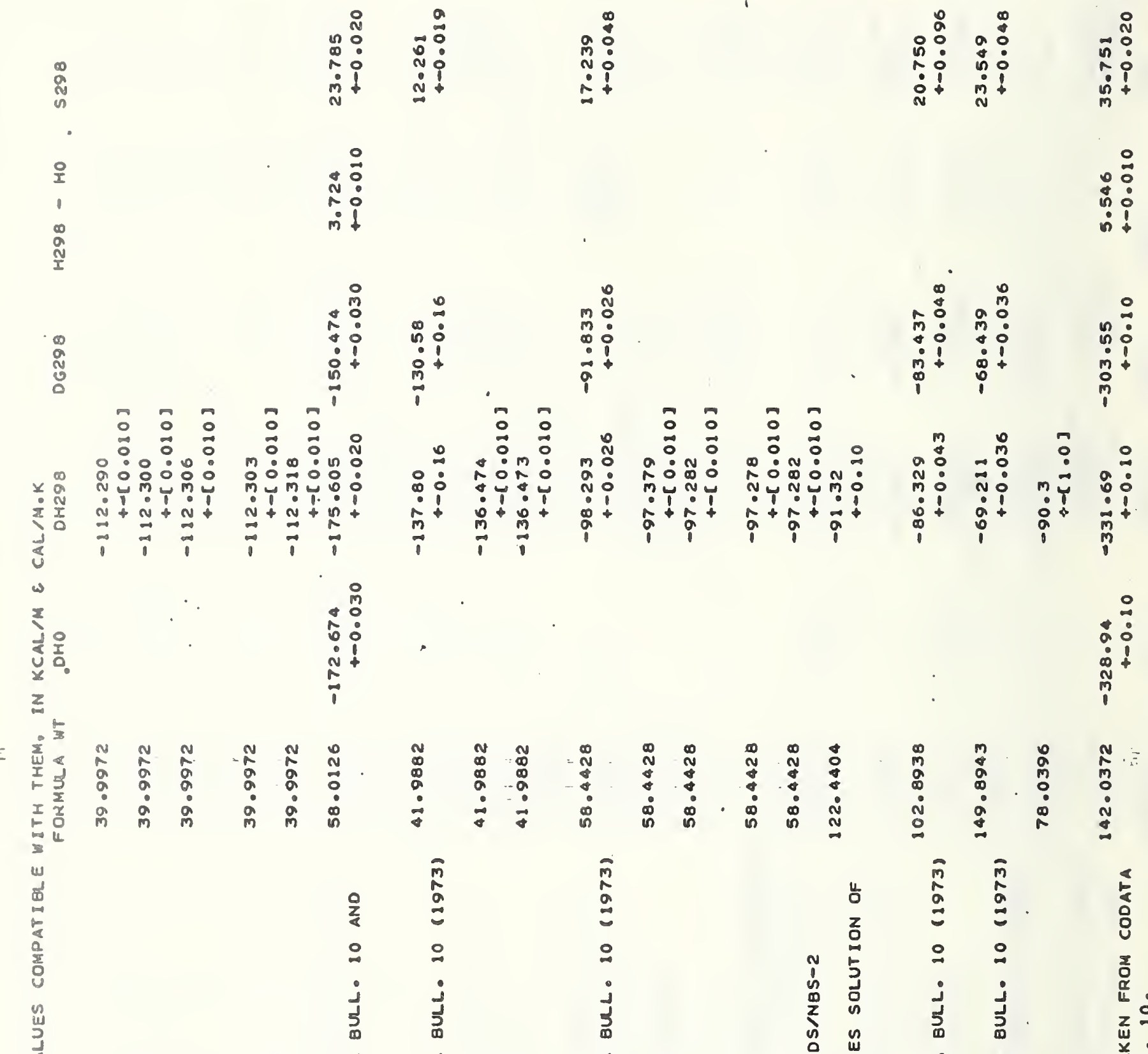

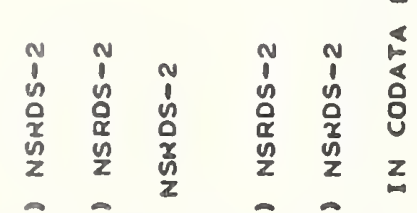

」

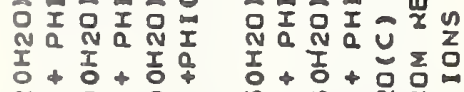

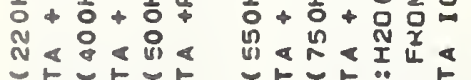

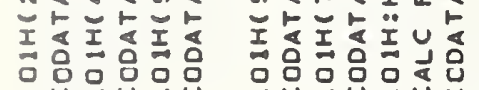

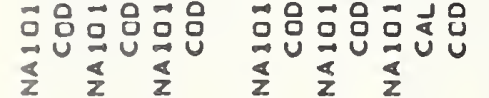

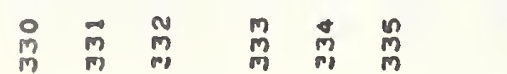

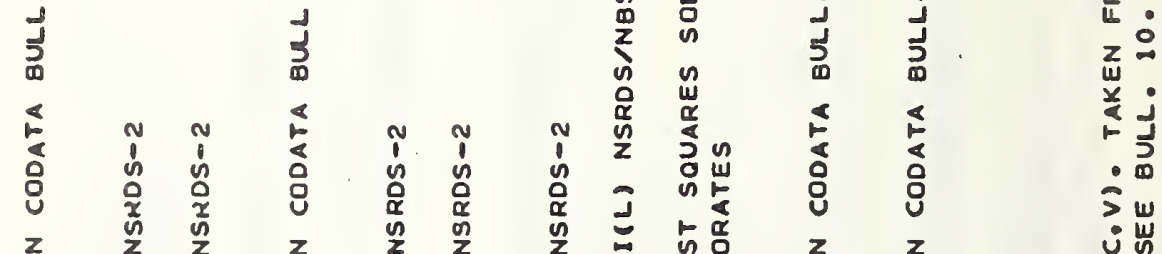

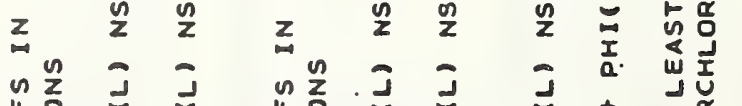

呟 J

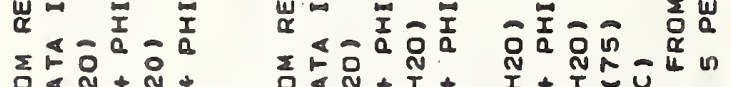

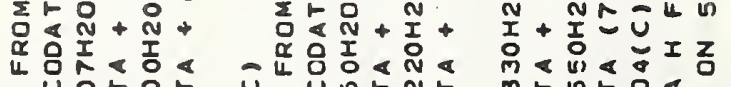

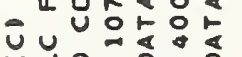

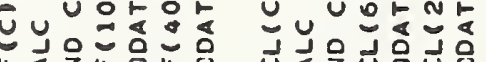

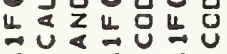

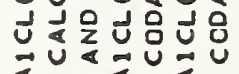

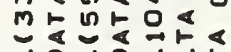

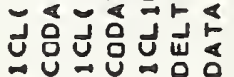

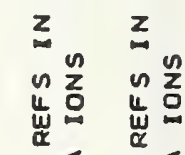

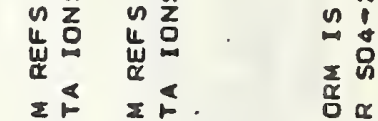

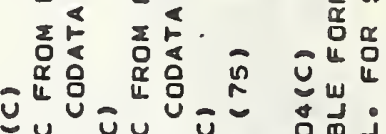
บّ̛

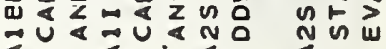
$\Sigma$ \& $\frac{8}{2}$

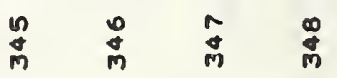

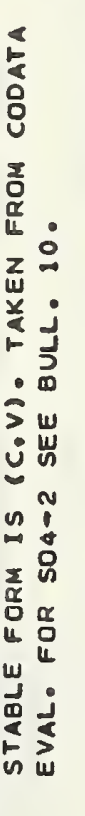

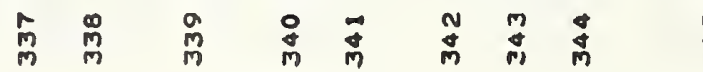

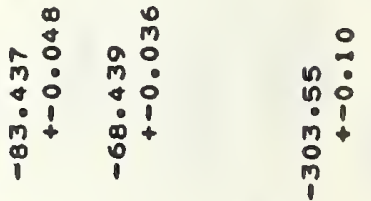

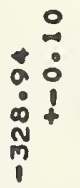



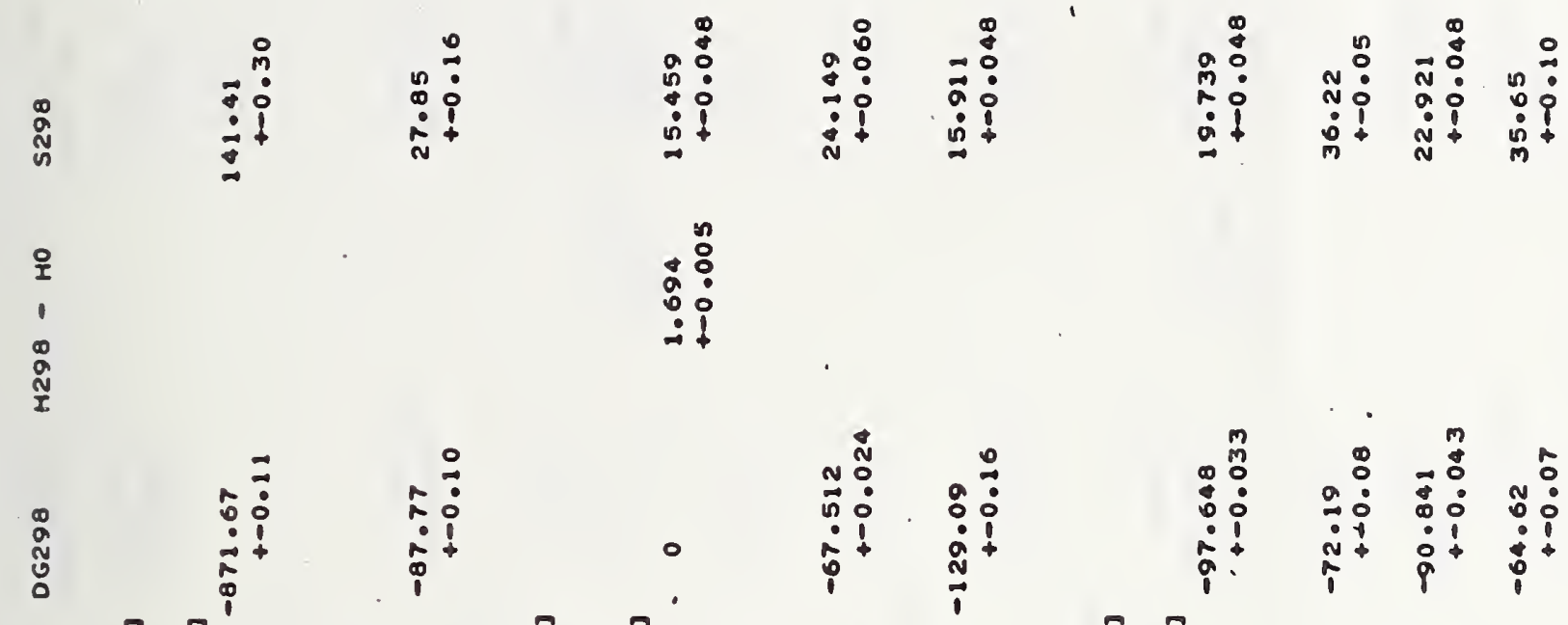

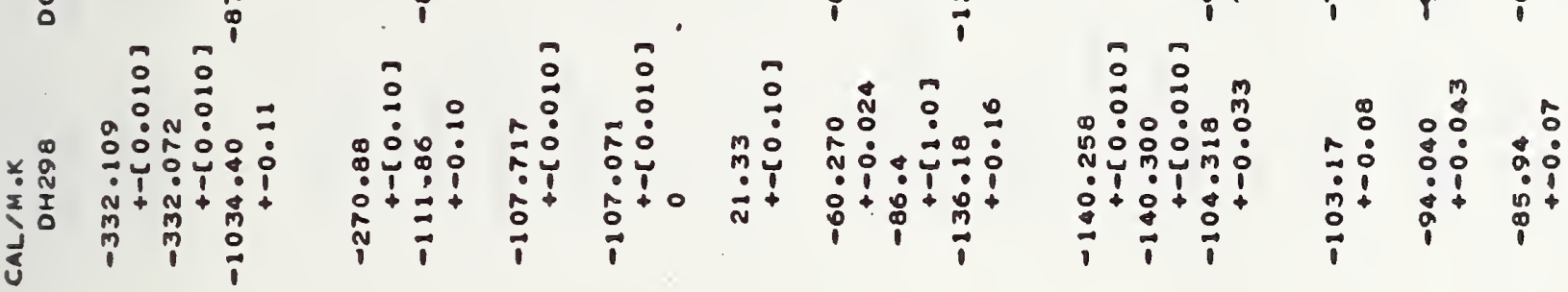

$\sum_{\substack{x \\ z}}^{\infty}$

3

占

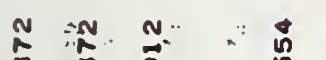

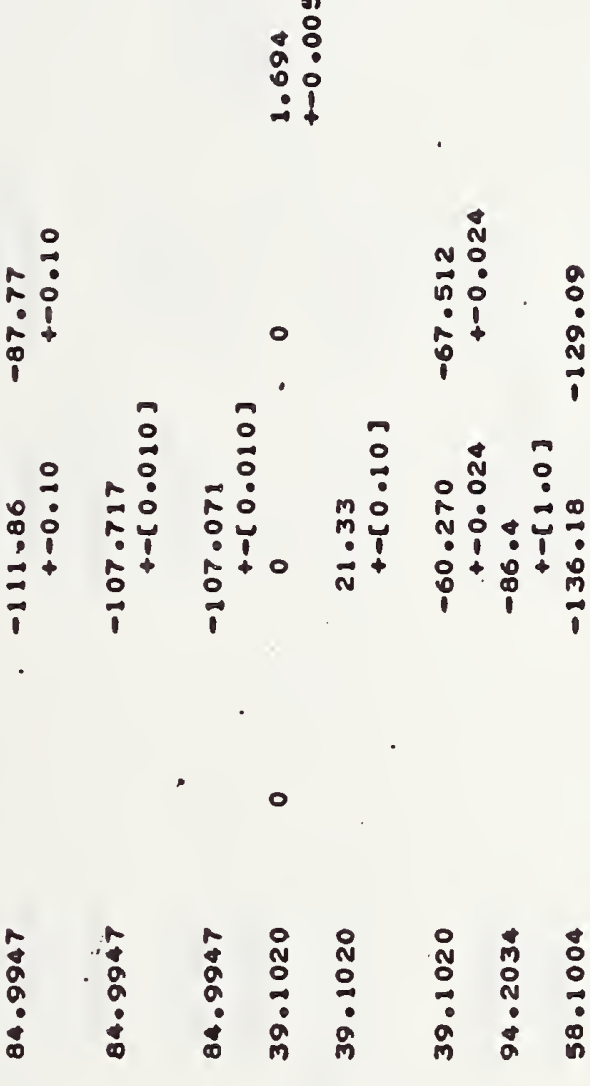

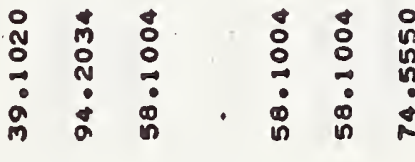

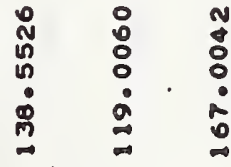

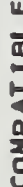

$\rightarrow$

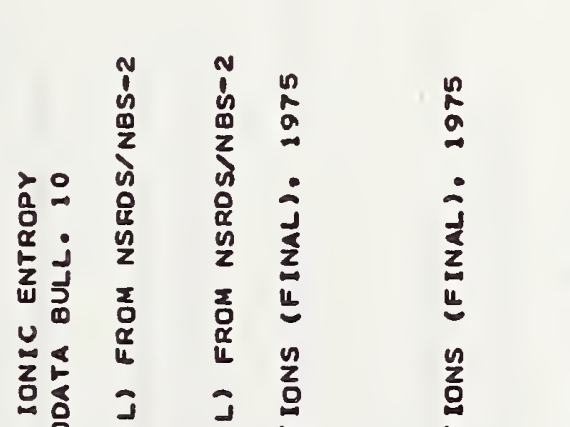

玄

$\stackrel{\text { a }}{a}$

$\therefore$

\a

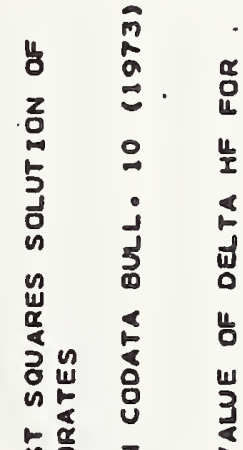

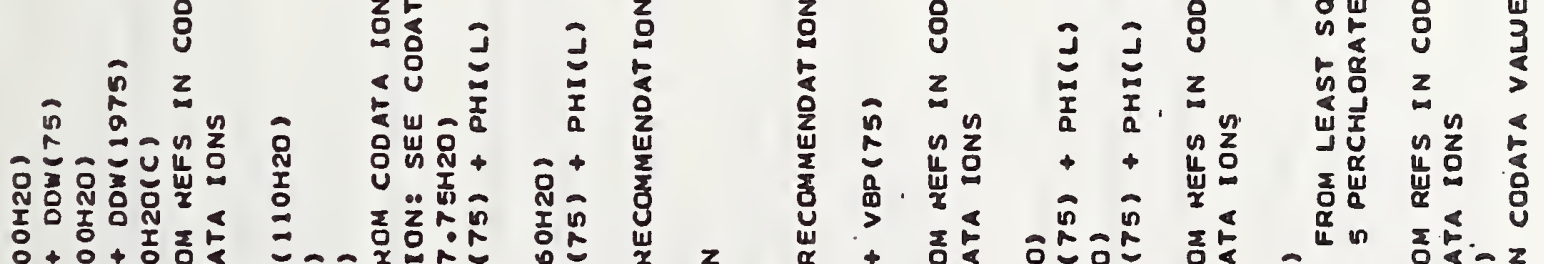

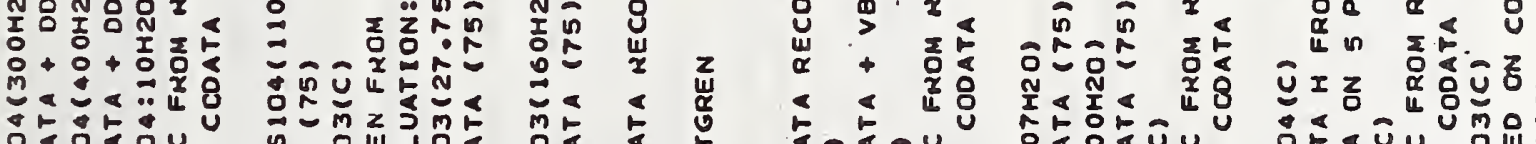

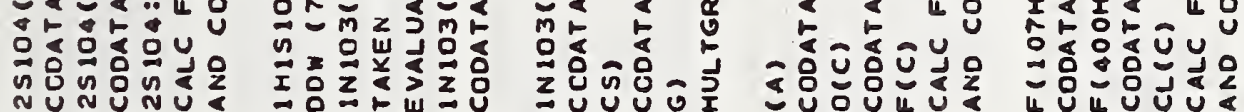

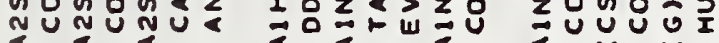
†U

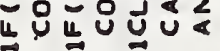
Ũz

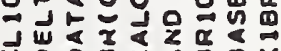

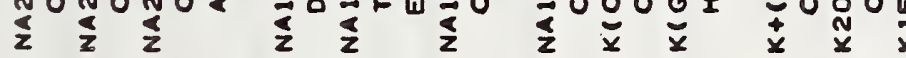

萬 

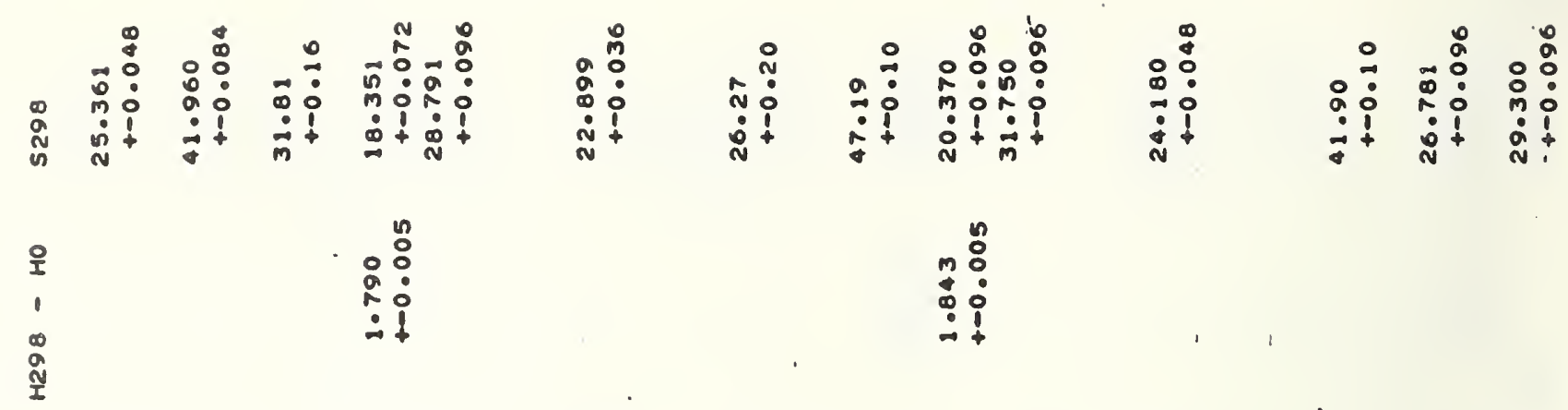

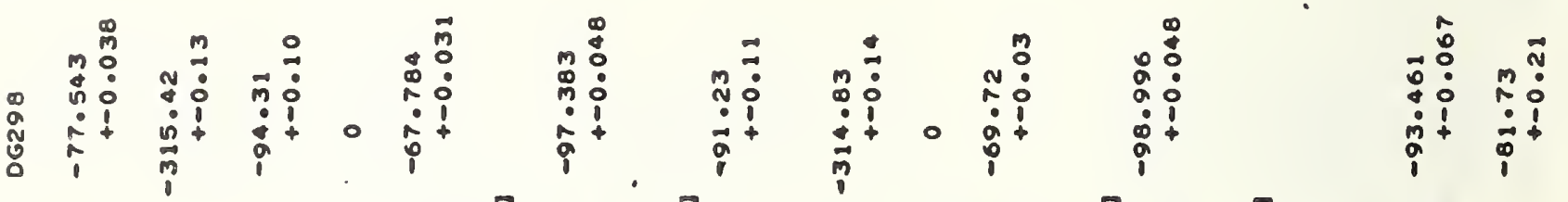

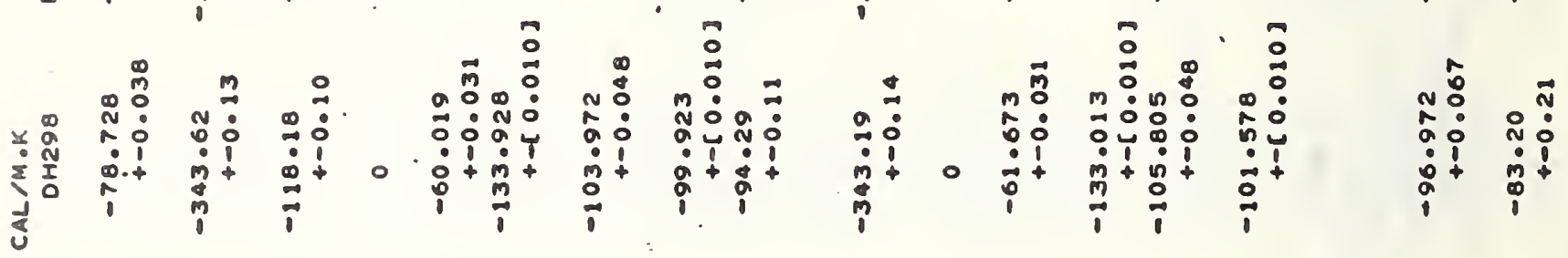

$\omega$

$x$
$y$
$y$

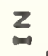

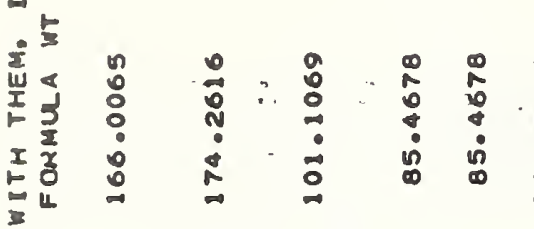

登

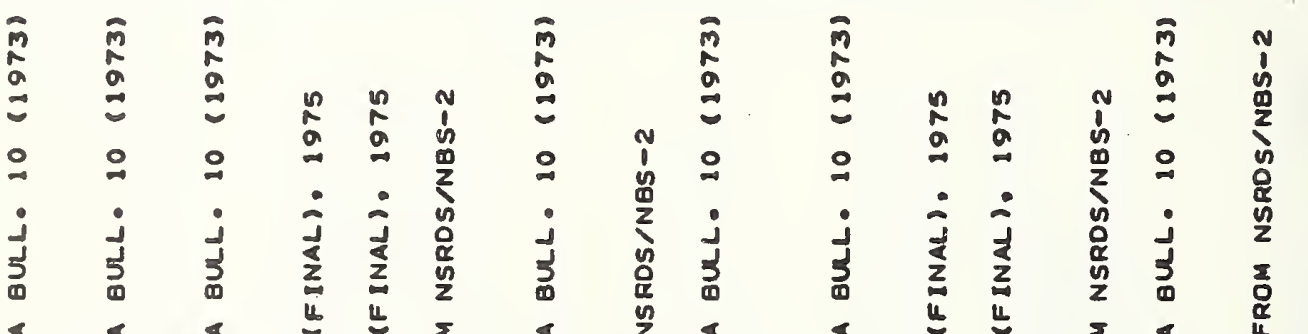

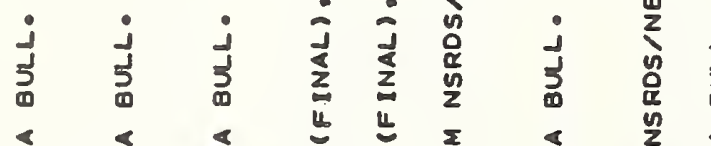

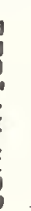


:

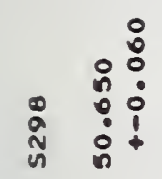

온

1

$\stackrel{\stackrel{9}{N}}{\text { I }}$

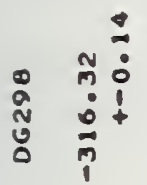

ن

$\omega$

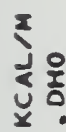

$z$

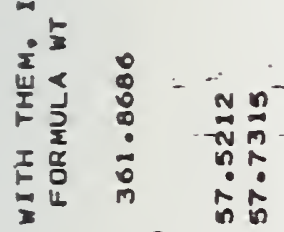

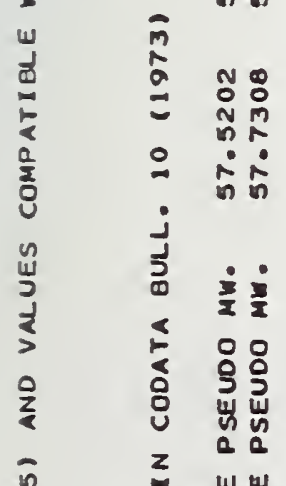

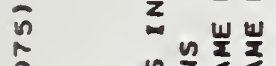

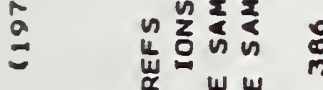

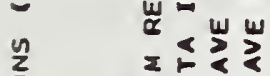

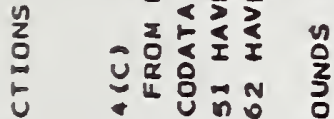

出

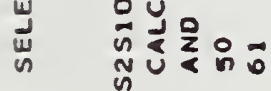

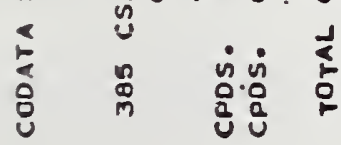




\begin{tabular}{|c|c|c|}
\hline \begin{tabular}{c|c} 
U.S. DEPT. OF COMM. & 1. PUBLICATION OR REPORT NO. \\
BIBLIOGRAPHIC DATA & NHEET
\end{tabular} & $\begin{array}{l}\text { 2. Gor't Accession } \\
\text { No. }\end{array}$ & 3. Recipient's Accession No. \\
\hline \multirow{2}{*}{\multicolumn{2}{|c|}{$\begin{array}{l}\text { 4. TITLE AND SUBTITLE } \\
\text { SELECTED THERMOCHEMICAL DATA COMPATIBLE WITH THE CODATA } \\
\text { RECOMMENDATIONS }\end{array}$}} & \multirow{2}{*}{$\begin{array}{l}\text { 5. Publication Date } \\
\text { January } 1976 \\
\text { 6. Performing Organization Code }\end{array}$} \\
\hline & & \\
\hline 7. AUTHOR(S) . B. Parker, D. D. Wagman, D. Garvin & & $\begin{array}{l}\text { 8. Performing Organ. Report No. } \\
\text { NBSIR } 75-968\end{array}$ \\
\hline \multirow{2}{*}{\multicolumn{2}{|c|}{$\begin{array}{l}\text { 9. PERFORMING ORGANIZATION NAME AND ADDRESS } \\
\qquad \begin{array}{l}\text { NATIONAL BUREAU OF STANDARDS } \\
\text { DEPARTMENT OF COMMERCE } \\
\text { WASHINGTON, D.C. } 20234\end{array}\end{array}$}} & $\begin{array}{l}\text { 10. Project/Task/Work Unit No. } \\
3165151\end{array}$ \\
\hline & & 11. Contract/Grant No. \\
\hline \multirow{2}{*}{\multicolumn{2}{|c|}{$\begin{array}{l}\text { 12. Sponsoring Organization Name and Complete Address (Street, City, State, ZIP) } \\
\text { Nationa } 1 \text { Bureau of Standards } \\
\text { Department of Commerce } \\
\text { Washington, DC } 20234\end{array}$}} & $\begin{array}{l}\text { 13. Type of Report \& Period } \\
\text { Covered } \\
\text { Interim }\end{array}$ \\
\hline & & 14. Sponsoring Agency Code \\
\hline
\end{tabular}

15. SUPPLEMENTARY NOTES

16. ABSTRACT (A 200-word or less lactual summary of most significant information. If document includes a significant bibliography or literature survey, mention it here.)

Selected thermochemica 1 properties data at $298.15 \mathrm{~K}$ are given for 384 substances. The data are compatible with the current recommendations of the CODATA Task Group on Key Values for Thermodynamics. The set of values provided here is suitable for use as auxiliary data in evaluations of the thermochemical properties of compounds of the actinide elements. Rules used in the conversion of units and the rounding of numbers are stated.

17. KEY WORDS (six to twelve entries; alphabetical order; capitalize anly the first letter of the first key word unless a proper name; separated by semicolons) CODATA compatible values; enthalpies of formation; entropy; gibbs energy of formation; selected thermodynamlc values; thermodynamic properties

18. AVAILABILITY X Unlimited

For Official Distribution. Do Not Release to NTIS

Order From Sup. of Doc., U.S. Government Printing Office Washington, D.C. 20402, SD Cat. No. C13

5 Order From National Technical Information Service (NTIS) Springfield, Virginia 22151

\begin{tabular}{|l|c|}
\hline $\begin{array}{l}\text { 19. SECURITY CLASS } \\
\text { (THIS REPURT) }\end{array}$ & 21. NO. OF PAGES \\
UNCL ASSIFIED & 34 \\
\hline $\begin{array}{l}\text { 20. SECURITY CLASS } \\
\text { (THIS PAGE) } \\
\text { UNCLASSIFIED }\end{array}$ & 22. Price \\
\hline
\end{tabular}


